\title{
On a Reduction of Nonlinear Partial Differential Equations of Briot-Bouquet Type
}

\author{
Hidetoshi TAHARA
}

Sophia University

\begin{abstract}
Let $F(t, x, u, v)$ be a holomorphic function in a neighborhood of the origin of $\mathbf{C}^{4}$ satisfying $F(0, x, 0,0) \equiv 0$ and $(\partial F / \partial v)(0, x, 0,0) \equiv 0$; then the equation (A) $t \partial u / \partial t=F(t, x, u, \partial u / \partial x)$ is called a partial differential equation of Briot-Bouquet type with respect to $t$, and the function $\lambda(x)=(\partial F / \partial u)(0, x, 0,0)$ is called the characteristic exponent. In [15], it is proved that if $\lambda(0) \notin(-\infty, 0] \cup\{1,2, \ldots\}$ holds the equation (A) is reduced to the simple form $\left(\mathrm{B}_{1}\right) t \partial w / \partial t=\lambda(x) w$. The present paper considers the case $\lambda(0)=K \in\{1,2, \ldots\}$ and proves the following result: if $\lambda(0)=K \in\{1,2, \ldots\}$ holds the equation (A) is reduced to the form ( $\left.\mathrm{B}_{2}\right)$ $t \partial w / \partial t=\lambda(x) w+\gamma(x) t^{K}$ for some holomorphic function $\gamma(x)$. The reduction is done by considering the coupling of two equations $(\mathrm{A})$ and $\left(\mathrm{B}_{2}\right)$, and by solving their coupling equations. The result is applied to the problem of finding all the holomorphic and singular solutions of $(\mathrm{A})$.
\end{abstract}

\section{Introduction}

Let $(t, x)$ be the variables in $\mathbf{C}_{t} \times \mathbf{C}_{x}$, and let $F(t, x, u, v)$ be a function defined in a polydisk $\Delta$ centered at the origin of $\mathbf{C}_{t} \times \mathbf{C}_{x} \times \mathbf{C}_{u} \times \mathbf{C}_{v}$. In this paper, we will consider the following nonlinear first order partial differential equation

$$
t \frac{\partial u}{\partial t}=F\left(t, x, u, \frac{\partial u}{\partial x}\right)
$$

under the assumptions

$\left.\mathrm{A}_{1}\right) \quad F(t, x, u, v)$ is holomorphic in $\Delta$,

A $) \quad F(0, x, 0,0) \equiv 0$ in $\Delta_{0}=\Delta \cap\{t=0, u=0, v=0\}$, and

A $) \frac{\partial F}{\partial v}(0, x, 0,0) \equiv 0$ in $\Delta_{0}$.

Then, the equation (1.1) is called a partial differential equation of Briot-Bouquet type with respect to $t$ (by Gérard-Tahara [9]): this is a natural generalization of Briot-Bouquet's ordinary

Received October 18, 2012; revised April 10, 2013 2010 Mathematics Subject Classification: 35A20

Key words and phrases: coupling equation, Briot-Bouquet type PDE, reduction, singular solution Supported by the Grant-in-Aid for Scientific Research No. 22540206 of Japan Society for the Promotion of Science. 
differential equation (see Briot-Bouquet [4], Hille [11] and Gérard [8]). The function

$$
\lambda(x)=\frac{\partial F}{\partial u}(0, x, 0,0)
$$

is called the characteristic exponent (or the characteristic exponent function) of (1.1).

We will consider the equation (1.1) in the space of holomorphic functions or in the class $\mathscr{S}_{\text {zero }}$ or $\mathscr{S}_{+}$defined below.

We denote by $\mathcal{R}(\mathbf{C} \backslash\{0\})$ the universal covering space of $\mathbf{C} \backslash\{0\}$. For $\theta>0$ and $r>0$ we write $S_{\theta}(r)=\{t \in \mathcal{R}(\mathbf{C} \backslash\{0\}) ;|\arg t|<\theta, 0<|t|<r\}$, and for $R>0$ we write $D_{R}=\{x \in \mathbf{C} ;|x| \leq R\}$. We denote by $\mathscr{S}_{\text {zero }}$ (resp. $\mathscr{S}_{+}$) the set of all holomorphic functions $u(t, x)$ on $S_{\theta}(r) \times D_{R}$ (for some $\theta>0, r>0$ and $R>0$ which may depend on $u(t, x)$ ) satisfying $|u(t, x)|=o(1)$ (as $t \longrightarrow 0$ in $S_{\theta}(r)$ ) uniformly on $D_{R}$ (resp. $|u(t, x)|=O\left(t^{a}\right)$ (as $t \longrightarrow 0$ in $S_{\theta}(r)$ ) uniformly on $D_{R}$ for some $a>0$ ). We note: $\mathscr{S}_{+} \subset \mathscr{S}_{\text {zero }}$ holds.

Baouendi-Goulaouic [2] first treated this type of equations in a little bit special form; then Gérard-Tahara [10] treated it in the general case and determined all the singular solutions belonging to the class $\mathscr{S}_{+}$in the case $\lambda(0) \notin \mathbf{N}^{*}$, and Yamazawa [16] solved the case $\lambda(0) \in$ $\mathbf{N}^{*}$. Later, Tahara [15] determined all the singular solutions belonging to the class $\mathscr{I}_{\text {zero }}$ by using the following reduction theorem for the equation (1.1):

THEOREM 1.1 ([15]). If $\left.\left.\left.\mathrm{A}_{1}\right), \mathrm{A}_{2}\right), \mathrm{A}_{3}\right)$ and $\lambda(0) \notin(-\infty, 0] \cup\{1,2, \ldots\}$ hold, the following two equations are equivalent (in the sense of Definition 2.5 in $\S 2$ ):

$$
\begin{aligned}
& t \frac{\partial u}{\partial t}=F\left(t, x, u, \frac{\partial u}{\partial x}\right) \text { in } \mathscr{S}_{\text {zero }}, \\
& t \frac{\partial w}{\partial t}=\lambda(x) w \text { in } \mathscr{S}_{\text {zero }} .
\end{aligned}
$$

Similar equations are also treated in Chen-Tahara [7], Chen-Luo-Tahara [5], Chen-LuoZhang [6], Luo-Chen-Zhang [13] and Lope-Roque-Tahara [12].

In this paper, we will consider the equation (1.1) of Briot-Bouquet type from the standpoint of transformation theory in the following case

$$
\lambda(0)=K \in\{1,2, \ldots\}:
$$

in this case we have

$$
\begin{aligned}
& |i+\lambda(x)(j-1)| \geq \sigma_{0}(i+j) \quad \text { on } D_{R} \\
& \quad \text { for any }(i, j) \in \mathbf{N} \times \mathbf{N} \backslash\{(0,0),(0,1),(K, 0)\}
\end{aligned}
$$

for some $\sigma_{0}>0$ and $R>0$. But, at $(i, j)=(K, 0)$ we have $|i+\lambda(0)(j-1)|=0$. This means that the operator $\tau_{k, d}-\lambda(x)$ with

$$
\tau_{k, d}=t \frac{\partial}{\partial t}+\sum_{0 \leq m \leq k-1} \lambda(x) u_{m} \frac{\partial}{\partial u_{m}}
$$


(where $k \in\{1,2, \ldots\}$ and $d$ indicates "diagonal") satisfies the Poincaré condition, but it has a resonance relation $i+\left(j_{1}+\cdots+j_{m}\right) \lambda(0)=\lambda(0)$ at $\left(i, j_{1}, \ldots, j_{m}\right)=(K, 0, \ldots, 0)$ and $x=0$. This operator $\tau_{k, d}-\lambda(x)$ will play an important role in our discussion.

About vector fields with Poincaré condition or with resonances, one can refer to Arnold [1], Yoshino [17], Björk [3] etc.

The following result is the main theorem of this paper.

THEOREM 1.2 (Main theorem). If the conditions $\left.\left.\left.\mathrm{A}_{1}\right), \mathrm{A}_{2}\right), \mathrm{A}_{3}\right)$ and (1.3) hold, we can find a holomorphic function $\gamma(x)$ in a neighborhood of $0 \in \mathbf{C}_{x}$ such that the following two equations are equivalent (in the sense of Definition 2.5 in $\$ 2$ ):

$$
\begin{aligned}
& t \frac{\partial u}{\partial t}=F\left(t, x, u, \frac{\partial u}{\partial x}\right) \quad \text { in } \mathscr{S}_{\text {zero }}, \\
& t \frac{\partial w}{\partial t}=\lambda(x) w+\gamma(x) t^{K} \quad \text { in } \mathscr{S}_{\text {zero }} .
\end{aligned}
$$

This yields the structure of singular solutions in $\mathscr{I}_{\text {zero }}$ (see Theorem 6.2 in $§ 6$ ).

The proof of Theorem 1.2 will be done by considering the coupling of two equations (1.6) and (1.7), and by solving their coupling equations.

The paper is organized as follows. In the next section 2, we will recall the formal theory of coupling of two partial differential equations developed in [14] and [15]. In section 3 we will look for formal solutions of the coupling equations, in section 4 we will give estimates of these formal solutions, and in section 5 we will complete the proof of Theorem 1.2. In the last section 6, as an application of Theorem 1.2, we will determine all the holomorphic and singular solutions of (1.1).

In the discussion of this paper, we write

$$
\begin{aligned}
& F(t, x, u, v)=a(x) t+\lambda(x) u+\sum_{i+j+\alpha \geq 2} c_{i, j, \alpha}(x) t^{i} u^{j} v^{\alpha}, \\
& R_{2}(t, x, u, v)=\sum_{i+j+\alpha \geq 2} c_{i, j, \alpha}(x) t^{i} u^{j} v^{\alpha}, \\
& R^{(p)}(t, x, u, v)=\sum_{i+j+\alpha=p} c_{i, j, \alpha}(x) t^{i} u^{j} v^{\alpha}, \quad p \geq 2 .
\end{aligned}
$$

By taking $R>0$ sufficiently small, we may suppose that $a(x), \lambda(x)$ and $c_{i, j, \alpha}(x)(i+j+\alpha \geq$ 2) are all holomorphic in a neighborhood of $D_{R}$.

\section{Formal theory of coupling equations}

In this section, we will recall the formal theory of the coupling of two partial differential equations. For details, see [14] and [15]. 
Let $F(t, x, u, v)$ and $G(t, x, u, v)$ be holomorphic functions in a neighborhood of $(0,0,0,0) \in \mathbf{C}_{t} \times \mathbf{C}_{x} \times \mathbf{C}_{u} \times \mathbf{C}_{v}$. We will consider the two equations

$$
\begin{aligned}
& t \frac{\partial u}{\partial t}=F\left(t, x, u, \frac{\partial u}{\partial x}\right), \\
& t \frac{\partial w}{\partial t}=G\left(t, x, w, \frac{\partial w}{\partial x}\right)
\end{aligned}
$$

in the framework of holomorphic functions.

We denote by $(t, x, \vec{u})=\left(t, x, u_{0}, u_{1}, u_{2}, \ldots\right) \in \mathbf{C}_{t} \times \mathbf{C}_{x} \times \mathbf{C}_{u}^{\infty}$ the complex variables, by $\mathcal{H}_{k, R}\left[t, u_{0}, \ldots, u_{k-1}\right]$ the set of all homogeneous polynomials of degree $k$ with respect to the variables $\left(t, u_{0}, \ldots, u_{k-1}\right)$ with holomorphic coefficients in $x \in D_{R}$, and by $\mathcal{M}_{R}[t, \vec{u}]$ the set of all formal sum $\phi=\phi\left(t, x, u_{0}, u_{1}, u_{2}, \ldots\right)$ of the form

$$
\phi=\sum_{k \geq 1} \phi_{k}\left(t, x, u_{0}, \ldots, u_{k-1}\right) \in \sum_{k \geq 1} \mathcal{H}_{k, R}\left[t, u_{0}, \ldots, u_{k-1}\right] .
$$

2.1. Coupling equations. The coupling of two partial differential equations (A) and (B) means that we consider the following partial differential equation with infinitely many variables $\left(t, x, u_{0}, u_{1}, u_{2}, \ldots\right)$

$$
t \frac{\partial \phi}{\partial t}+\sum_{m \geq 0} D^{m}[F]\left(t, x, u_{0}, \ldots, u_{m+1}\right) \frac{\partial \phi}{\partial u_{m}}=G(t, x, \phi, D[\phi])
$$

(where $\phi=\phi\left(t, x, u_{0}, u_{1}, u_{2}, \ldots\right)$ is the unknown function), or the following partial differential equation with infinitely many variables $\left(t, x, w_{0}, w_{1}, w_{2}, \ldots\right)$

$$
t \frac{\partial \psi}{\partial t}+\sum_{m \geq 0} D^{m}[G]\left(t, x, w_{0}, \ldots, w_{m+1}\right) \frac{\partial \psi}{\partial w_{m}}=F(t, x, \psi, D[\psi])
$$

(where $\psi=\psi\left(t, x, w_{0}, w_{1}, w_{2}, \ldots\right)$ is the unknown function). In the equation $(\Phi)$ (resp. $(\Psi)$ ), the notation $D$ means the following vector field with infinite many variables

$$
D=\frac{\partial}{\partial x}+\sum_{i \geq 0} u_{i+1} \frac{\partial}{\partial u_{i}} \quad\left(\text { resp. } D=\frac{\partial}{\partial x}+\sum_{i \geq 0} w_{i+1} \frac{\partial}{\partial w_{i}}\right),
$$

and $D^{m}[F](m=0,1,2, \ldots)$ are defined by $D^{0}[F]=F, D[F]=D F, D^{2}[F]=D(D[F])$, $D^{3}[F]=D\left(D^{2}[F]\right)$ and so on. These two equations $(\Phi)$ and $(\Psi)$ are called the coupling equations of $(\mathrm{A})$ and $(\mathrm{B})$.

The convenience of considering the coupling equation lies in the following proposition.

Proposition 2.1. (1) If $\phi\left(t, x, u_{0}, u_{1}, \ldots\right) \in \mathcal{M}_{R}[t, \vec{u}]$ is a solution of $(\Phi)$ and if $u(t, x)$ is a solution of $(\mathrm{A})$, then the function $w(t, x)=\phi(t, x, u, \partial u / \partial x, \ldots)$ is a solution of (B).

(2) If $\psi\left(t, x, w_{0}, w_{1}, \ldots\right) \in \mathcal{M}_{R}[t, \vec{w}]$ is a solution of $(\Psi)$ and if $w(t, x)$ is a solution of $(\mathrm{B})$, then the function $u(t, x)=\psi(t, x, w, \partial w / \partial x, \ldots)$ is a solution of (A). 
In order to understand the relation between $(\Phi)$ and $(\Psi)$, let us introduce the notion of reversibility.

Definition 2.2. Let $\phi\left(t, x, u_{0}, u_{1}, \ldots\right) \in \mathcal{M}_{R}[t, \vec{u}]$. We say that the relation $w=$ $\phi(t, x, u, \partial u / \partial x, \ldots)$ is reversible with respect to $u$ and $w$, if there is a $\psi\left(t, x, w_{0}, w_{1}, \ldots\right) \in$ $\mathcal{M}_{R}[t, \vec{w}]$ such that the relation

$$
w_{j}=D^{j}[\phi]\left(t, x, u_{0}, u_{1}, u_{2}, \ldots\right), \quad j=0,1,2, \ldots
$$

is equivalent to the relation

$$
u_{j}=D^{j}[\psi]\left(t, x, w_{0}, w_{1}, w_{2}, \ldots\right), \quad j=0,1,2, \ldots
$$

We call $\psi\left(t, x, w_{0}, w_{1}, w_{2}, \ldots\right)$ the reverse function of $\phi\left(t, x, u_{0}, u_{1}, u_{2} \ldots\right)$.

PROPOSITION 2.3. Let $\phi\left(t, x, u_{0}, u_{1}, \ldots\right) \in \mathcal{M}_{R}[t, \vec{u}]$, and suppose that the relation $w=\phi(t, x, u, \partial u / \partial x, \ldots)$ is reversible with respect to $u$ and $w$. If $\phi\left(t, x, u_{0}, u_{1}, \ldots\right)$ is a solution of $(\Phi)$, then the reverse function $\psi\left(t, x, w_{0}, w_{1}, \ldots\right)$ is a solution of $(\Psi)$.

2.2. Equivalence of $(\mathbf{A})$ and $(\mathbf{B})$. Let $\mathcal{F}$ and $\mathcal{G}$ be function-spaces in which we can consider the following two partial differential equations:

$$
\begin{aligned}
& t \frac{\partial u}{\partial t}=F\left(t, x, u, \frac{\partial u}{\partial x}\right) \quad \text { in } \mathcal{F}, \\
& t \frac{\partial w}{\partial t}=G\left(t, x, w, \frac{\partial w}{\partial x}\right) \quad \text { in } \mathcal{G} .
\end{aligned}
$$

Set

$$
\begin{aligned}
& \mathcal{S}_{A}=\text { the set of all solutions of [A] in } \mathcal{F}, \\
& \mathcal{S}_{B}=\text { the set of all solutions of [B] in } \mathcal{G} .
\end{aligned}
$$

Then, if the coupling equation $(\Phi)$ has a solution $\phi\left(t, x, u_{0}, u_{1}, \ldots\right)$ and if $\phi(t, x$, $u, \partial u / \partial x, \ldots) \in \mathcal{G}$ is well-defined for any $u \in \mathcal{S}_{A}$, we can define the mapping

$$
\Phi: \mathcal{S}_{A} \ni u(t, x) \longmapsto w(t, x)=\phi(t, x, u, \partial u / \partial x, \ldots) \in \mathcal{S}_{B} .
$$

If the relation $w=\phi(t, x, u, \partial u / \partial x, \ldots)$ is reversible with respect to $u$ and $w$, and if the reverse function $\psi\left(t, x, w_{0}, w_{1}, \ldots\right)$ satisfies $\psi(t, x, w, \partial w / \partial x, \ldots) \in \mathcal{F}$ for any $w \in \mathcal{S}_{B}$, we can also define the mapping

$$
\Psi: \mathcal{S}_{B} \ni w(t, x) \longmapsto u(t, x)=\psi(t, x, w, \partial w / \partial x, \ldots) \in \mathcal{S}_{A} .
$$

Set $w(t, x)=\phi(t, x, u, \partial u / \partial x, \ldots)$; then by the definition of $D$ we have

$$
(\partial / \partial x)^{j} w=D^{j}[\phi](t, x, u, \partial u / \partial x, \ldots), \quad j=0,1,2, \ldots
$$

Similarly, if we set $u(t, x)=\psi(t, x, w, \partial w / \partial x, \ldots)$ we have

$$
(\partial / \partial x)^{j} u=D^{j}[\psi](t, x, w, \partial w / \partial x, \ldots), \quad j=0,1,2, \ldots
$$


Since the relation $w=\phi(t, x, u, \partial u / \partial x, \ldots)$ is reversible with respect to $u$ and $w$, we see that the relation (2.4) and (2.5) are equivalent; therefore we have $\Psi \circ \Phi=$ identity in $\mathcal{S}_{A}$ and $\Phi \circ \Psi=$ identity in $\mathcal{S}_{B}$. Thus, we obtain

THEOREM 2.4. Suppose that the coupling equation $(\Phi)$ has a solution $\phi(t, x$, $\left.u_{0}, u_{1}, \ldots\right) \in \mathcal{M}_{R}[t, \vec{u}]$ and that the relation $w=\phi(t, x, u, \partial u / \partial x, \ldots)$ is reversible with respect to $u$ and $w$. If both mappings (2.2) and (2.3) are well-defined, we can conclude that the both mappings are bijective and one is the inverse of the other.

By this theorem, we may say:

Definition 2.5. (1) If the coupling equation $(\Phi)$ (resp. $(\Psi)$ ) has a solution $\phi\left(t, x, u_{0}, u_{1} \ldots\right) \in \mathcal{M}_{R}[t, \vec{u}]$ (resp. $\left.\psi\left(t, x, w_{0}, w_{1} \ldots\right) \in \mathcal{M}_{R}[t, \vec{w}]\right)$ and if the relation $w=\phi(t, x, u, \partial u / \partial x, \ldots)($ resp. $u=\psi(t, x, w, \partial w / \partial x, \ldots))$ is reversible with respect to $u$ and $w$ (or $w$ and $u$ ), then we say that the two equations (A) and (B) are formally equivalent.

(2) In addition, if both mappings (2.2) and (2.3) are well-defined, we say that the two equations $[\mathrm{A}]$ and $[\mathrm{B}]$ are equivalent.

2.3. A sufficient condition for the reversibility. As is seen above, the condition of the reversibility of $\phi\left(t, x, u_{0}, u_{1}, \ldots\right)$ is very important. The following proposition gives a sufficient condition.

Proposition 2.6. If $\phi\left(t, x, u_{0}, u_{1}, \ldots\right) \in \mathcal{M}_{R}[t, \vec{u}]$ satisfies $\left(\partial \phi / \partial u_{0}\right)(0, x, 0$, $0, \ldots) \neq 0$ on $D_{R}$, the relation $w=\phi(t, x, u, \partial u / \partial x, \ldots)$ is reversible with respect to $u$ and $w$, and the reverse function $\psi\left(t, x, w_{0}, w_{1}, \ldots\right) \in \mathcal{M}_{R}[t, \vec{w}]$ also satisfies $\left(\partial \psi / \partial w_{0}\right)(0, x, 0,0, \ldots) \neq 0$ on $D_{R}$.

\section{Formal solutions of $(\Phi)$ and $(\Psi)$}

Now, let us return to the situation in Theorem 1.2. We let $F(t, x, u, v)$ be a holomorphic function in a neighborhood of $(0,0,0,0) \in \mathbf{C}_{t} \times \mathbf{C}_{x} \times \mathbf{C}_{u} \times \mathbf{C}_{v}$ satisfying $\left.\left.\left.\mathrm{A}_{1}\right), \mathrm{A}_{2}\right), \mathrm{A}_{3}\right)$ and (1.3).

In this section, we will find formal solutions of our coupling equations

$$
\begin{aligned}
& t \frac{\partial \phi}{\partial t}+\sum_{m \geq 0} D^{m}[F]\left(t, x, u_{0}, \ldots, u_{m+1}\right) \frac{\partial \phi}{\partial u_{m}}=\lambda(x) \phi+\gamma(x) t^{K}, \quad \text { and } \\
& t \frac{\partial \psi}{\partial t}+\sum_{m \geq 0}\left(\gamma^{(m)}(x) t^{K}+\sum_{0 \leq i \leq m} \lambda_{m, i}(x) w_{i}\right) \frac{\partial \psi}{\partial w_{m}}=F(t, x, \psi, D[\psi]),
\end{aligned}
$$

where $D$ is the vector field in (2.1) with infinitely many variables $\left(x, u_{0}, u_{1}, \ldots\right)$ (resp. $\left.\left(x, w_{0}, w_{1}, \ldots\right)\right), \gamma^{(m)}(x)=(\partial / \partial x)^{m} \gamma(x)(m=0,1,2, \ldots)$ and

$$
\lambda_{m, i}(x)=\frac{m !}{i !(m-i) !}\left(\frac{\partial}{\partial x}\right)^{m-i} \lambda(x), \quad 0 \leq i \leq m .
$$


The above form of $(\Psi)$ comes from the form of $(\Psi)$ in $\S 2$ and the following formula:

$$
D^{m}\left[\gamma(x) t^{K}+\lambda(x) w_{0}\right]=\gamma^{(m)}(x) t^{K}+\sum_{0 \leq i \leq m} \lambda_{m, i}(x) w_{i} .
$$

3.1. Preliminary calculations. In the first step of our consideration, the function $\gamma(x)$ is unknown, and so for a while, instead of treating the equations $(\Phi)$ and $(\Psi)$, we will consider the following two equations

$$
t \frac{\partial \phi}{\partial t}+\sum_{m \geq 0} D^{m}[F]\left(t, x, u_{0}, \ldots, u_{m+1}\right) \frac{\partial \phi}{\partial u_{m}}=\lambda(x) \phi+\gamma_{1}(x) t^{K}
$$

(where $\left(t, x, u_{0}, u_{1}, \ldots\right)$ is the variables, and $\phi=\phi\left(t, x, u_{0}, u_{1}, \ldots\right)$ and $\gamma_{1}(x)$ are the unknown functions), and

$$
t \frac{\partial \psi}{\partial t}+\sum_{m \geq 0}\left(\gamma_{2}^{(m)}(x) t^{K}+\sum_{0 \leq i \leq m} \lambda_{m, i}(x) w_{i}\right) \frac{\partial \psi}{\partial w_{m}}=F(t, x, \psi, D[\psi])
$$

(where $\left(t, x, w_{0}, w_{1}, \ldots\right)$ is the variables, and $\psi=\psi\left(t, x, w_{0}, w_{1}, \ldots\right)$ and $\gamma_{2}(x)$ are the unknown functions).

For $R>0$ we denote by $\mathcal{O}_{R}$ the set of all holomorphic functions in a neighborhood of $D_{R}$. By a calculation we can see:

Proposition 3.1. Suppose the conditions $\left.\left.\left.\mathrm{A}_{1}\right), \mathrm{A}_{2}\right), \mathrm{A}_{3}\right)$ and $\lambda(0)=K \in\{1,2, \ldots\}$. Let $R>0$ be sufficiently small. Then we have:

(1) For any $X(x) \in \mathcal{O}_{R}$ there uniquely exists a $\gamma_{1}(x) \in \mathcal{O}_{R}$ such that the equation (3.1) has a unique formal solution $\phi\left(t, x, u_{0}, u_{1}, \ldots\right)$ of the form

$$
\phi=u_{0}+\cdots+X(x) t^{K}+\cdots \in \sum_{k \geq 1} \mathcal{H}_{k, R}\left[t, u_{0}, \ldots, u_{k-1}\right]
$$

with the condition that the coefficient of $u_{0}$ is equal to 1 and the coefficient of $t^{K}$ is equal to $X(x)$. Moreover, $\gamma_{1}(x)$ is expressed in the form $\gamma_{1}(x)=(K-\lambda(x)) X(x)+\mu_{1}(x)$ for some $\mu_{1}(x) \in \mathcal{O}_{R}$ which depends only on $F(t, x, u, v)$ and not on the choice of $X(x)$.

(2) For any $Y(x) \in \mathcal{O}_{R}$ there uniquely exists a $\gamma_{2}(x) \in \mathcal{O}_{R}$ such that the equation (3.2) has a unique formal solution $\psi\left(t, x, w_{0}, w_{1}, \ldots\right)$ of the form

$$
\psi=w_{0}+\cdots+Y(x) t^{K}+\cdots \in \sum_{k \geq 1} \mathcal{H}_{k, R}\left[t, w_{0}, \ldots, w_{k-1}\right]
$$

with the condition that the coefficient of $w_{0}$ is equal to 1 and the coefficient of $t^{K}$ is equal to $Y(x)$. Moreover, $\gamma_{2}(x)$ is expressed in the form $\gamma_{2}(x)=-(K-\lambda(x)) Y(x)+\mu_{2}(x)$ for some $\mu_{2}(x) \in \mathcal{O}_{R}$ which depends only on $F(t, x, u, v)$ and not on the choice of $Y(x)$. 
Proof. In the case $K=1$, if we set $\mu_{1}(x)=a(x), \gamma_{1}(x)=(1-\lambda(x)) X(x)+a(x)$, $\mu_{2}(x)=a(x)$ and $\gamma_{2}(x)=-(1-\lambda(x)) Y(x)+a(x)$, we can show Proposition 3.1 by the same calculations as in [\$\$3.1 and 3.2 of [15]].

Let us show the case $K \geq 2$. Suppose $K \geq 2$. First, let us look for a formal solution of the equation (3.1) in the form

$$
\phi=\sum_{k \geq 1} \phi_{k}\left(t, x, u_{0}, \ldots, u_{k-1}\right) \in \sum_{k \geq 1} \mathcal{H}_{k, R}\left[t, u_{0}, \ldots, u_{k-1}\right] .
$$

with $\left(\partial \phi_{1} / \partial u_{0}\right)=1$. As in the last part of $\S 1$, we set

$$
\begin{aligned}
& F\left(t, x, u_{0}, u_{1}\right)=a(x) t+\lambda(x) u_{0}+R_{2}\left(t, x, u_{0}, u_{1}\right), \\
& R_{2}\left(t, x, u_{0}, u_{1}\right)=\sum_{p \geq 2} R^{(p)}\left(t, x, u_{0}, u_{1}\right) \in \sum_{p \geq 2} \mathcal{H}_{p, R}\left[t, u_{0}, u_{1}\right], \\
& \tau_{k}=t \frac{\partial}{\partial t}+\sum_{0 \leq m \leq k-1}\left(a^{(m)}(x) t+\sum_{0 \leq i \leq m} \lambda_{m, i}(x) u_{i}\right) \frac{\partial}{\partial u_{m}}, \quad k \geq 1 .
\end{aligned}
$$

This vector field $\tau_{k}$ is expressed in the form

$$
\left(t, u_{0}, \ldots, u_{k-1}\right) A_{k}{ }^{t}\left(\partial / \partial t, \partial / \partial u_{0}, \ldots, \partial / \partial u_{k-1}\right)
$$

(where $t$ of ${ }^{t}\left(x_{0}, x_{1}, \ldots, x_{k-1}\right)$ denotes the transposed of the vector) for some upper triangular matrix $A_{k}$ : the vector field corresponding to the diagonal part of this matrix $A_{k}$ is just the one in (1.5) and so the operator $\tau_{k}-\lambda(x)$ satisfies the Poincaré condition. Our equation (3.1) is decomposed into the following recurrent formulas:

$$
\left(\tau_{1}-\lambda(x)\right) \phi_{1}=0 \quad \text { in } \mathcal{H}_{1, R}\left[t, u_{0}\right]
$$

and for $k \geq 2$

$(3.4)_{k}\left(\tau_{k}-\lambda(x)\right) \phi_{k}$

$$
=\delta_{k, K} \gamma_{1}(x) t^{K}-\sum_{1 \leq q \leq k-1} \sum_{0 \leq m \leq q-1} D^{m}\left[R^{(k-q+1)}\right]\left(t, x, u_{0}, \ldots, u_{m+1}\right) \frac{\partial \phi_{q}}{\partial u_{m}}
$$

in $\mathcal{H}_{k, R}\left[t, u_{0}, \ldots, u_{k-1}\right]$, where $\delta_{k, K}$ denotes the Kronecker's delta.

Since $|i+\lambda(x)(j-1)| \geq \sigma(i+j)$ on $D_{R}$ holds for any $(i, j) \in \mathbf{N} \times \mathbf{N} \backslash\{(0,0)$, $(0,1),(K, 0)\}$ (see (1.4)), in the case $k \neq K$ we can solve (3.4) $k$ inductively on $k$ by using the following lemma:

Lemma 3.2 (Lemma 3.1.3 in [15]). (1) If $\lambda(x) \neq 1$ on $D_{R}$, the equation $\left(\tau_{1}-\right.$ $\lambda(x)) \phi_{1}=0$ has a solution $\phi_{1} \in \mathcal{H}_{1, R}\left[t, u_{0}\right]$ of the form

$$
\phi_{1}=\frac{-a(x) \beta(x)}{1-\lambda(x)} t+\beta(x) u_{0}
$$

and $\beta(x) \in \mathcal{O}_{R}$ can be chosen arbitrarily. 
(2) Let $k \geq 2$. If $|i+\lambda(x)(j-1)| \neq 0$ on $D_{R}$ for any $(i, j) \in \mathbf{N} \times \mathbf{N}$ with $i+j=k$, then for any $f_{k} \in \mathcal{H}_{k, R}\left[t, u_{0}, \ldots, u_{k-1}\right]$ the equation $\left(\tau_{k}-\lambda(x)\right) \phi_{k}=f_{k}$ has a unique solution $\phi_{k} \in \mathcal{H}_{k, R}\left[t, u_{0}, \ldots, u_{k-1}\right]$.

Thus, the problem lies only in the treatment of (3.4) $)_{K}$. In the case $K \geq 2$, the terms $\phi_{1}=u_{0}+\left(-a(x) /(1-\lambda(x)) t\right.$ and $\phi_{k}\left(t, x, u_{0}, \ldots, u_{k-1}\right)(2 \leq k \leq K-1)$ are determined by Lemma 3.2, and $(3.4)_{K}$ is written as

$$
\begin{aligned}
\left(\tau_{K}-\right. & \lambda(x)) \phi_{K} \\
& =\gamma_{1}(x) t^{K}-\sum_{1 \leq q \leq K-1} \sum_{0 \leq m \leq q-1} D^{m}\left[R^{(K-q+1)}\right]\left(t, x, u_{0}, \ldots, u_{m+1}\right) \frac{\partial \phi_{q}}{\partial u_{m}}:
\end{aligned}
$$

we note that $\phi_{q}(1 \leq q \leq K-1)$ are already known. If we write

$$
\phi_{K}=X(x) t^{K}+\sum_{i+|j|=K,(i,|j|) \neq(K, 0)} \phi_{i, j}(x) t^{i} u_{0}^{j_{0}} \cdots u_{k-1} j_{k-1}
$$

(where $j=\left(j_{0}, j_{1}, \ldots, j_{k-1}\right)$ and $\left.|j|=j_{0}+j_{1}+\cdots+j_{k-1}\right)$, by a calculation we see that $\left\{\phi_{i, j}(x) \in \mathcal{O}_{R} ; i+|j|=K,(i,|j|) \neq(K, 0)\right\}$ are also uniquely determined and they are independent of $X(x)$ and $\gamma_{1}(x)$. If these $\phi_{i, j}(x)(i+|j|=K$ and $|j| \geq 1)$ are known, by comparing the coefficient of $t^{K}$ in the both sides of (3.5) we have the equation

$$
\begin{aligned}
(K-\lambda(x)) X(x)+\sum_{0 \leq m \leq K-1} a^{(m)}(x) \phi_{K-1, e_{m}}(x) \\
=\gamma_{1}(x)-\sum_{1 \leq q \leq K-1} \sum_{0 \leq m \leq q-1} c_{K-q+1,0,0}^{(m)}(x) \phi_{q-1, e_{m}}(x)
\end{aligned}
$$

under the following notations: (1) $e_{m}$ in $\phi_{K-1, e_{m}}(x)$ is $e_{m}=\left(j_{0}, j_{1}, \ldots, j_{K-1}\right)$ with $j_{m}=1$ and $j_{p}=0$ for $p \neq m$, (2) $\phi_{K-1, e_{m}}(x)$ is the coefficient of $t^{K-1} u_{m}$ in $\phi_{K}\left(t, x, u_{0}, \ldots, u_{K-1}\right)$, (3) $e_{m}$ in $\phi_{q-1, e_{m}}(x)$ is $e_{m}=\left(j_{0}, j_{1}, \ldots, j_{q-1}\right)$ with $j_{m}=1$ and $j_{p}=0$ for $p \neq m$, (4) $\phi_{q-1, e_{m}}(x)$ is the coefficient of $t^{q-1} u_{m}$ in $\phi_{q}\left(t, x, u_{0}, \ldots, u_{q-1}\right)$, and (5) $c_{K-q+1,0,0}^{(m)}(x) t^{K-q+1}=\left(D^{m}\left[R^{(K-q+1)}\right]\right)(t, x, 0, \ldots, 0)$. Note that $c_{K-q+1,0,0}(x)$ is just the coefficient of $t^{K-q+1}$ in the Taylor expansion of $R_{2}\left(t, x, u_{0}, u_{1}\right)$ with respect to $\left(t, u_{0}, u_{1}\right)$.

Therefore, if we set

$$
\begin{aligned}
\gamma_{1}(x)= & (K-\lambda(x)) X(x)+\sum_{0 \leq m \leq K-1} a^{(m)}(x) \phi_{K-1, e_{m}}(x) \\
& +\sum_{1 \leq q \leq K-1} \sum_{0 \leq m \leq q-1} c_{K-q+1,0,0}^{(m)}(x) \phi_{q-1, e_{m}}(x)
\end{aligned}
$$

we have the relation (3.6); this yields (3.5) (that is, $(3.4)_{K}$ ).

For $k \geq K+1$, the situation is the same as in the non-resonant case, and so by Lemma 3.2 we can uniquely determine $\phi_{k}\left(t, x, u_{0}, \ldots, u_{k-1}\right) \in \mathcal{H}_{k, R}\left[t, u_{0}, \ldots, u_{k-1}\right]$ inductively 
on $k$ so that $(3.4)_{k}$ is satisfied for $k \geq K+1$. This proves the part (1), and we have

$$
\begin{aligned}
\mu_{1}(x)= & \sum_{0 \leq m \leq K-1} a^{(m)}(x) \phi_{K-1, e_{m}}(x) \\
& +\sum_{1 \leq q \leq K-1} \sum_{0 \leq m \leq q-1} c_{K-q+1,0,0}^{(m)}(x) \phi_{q-1, e_{m}}(x) .
\end{aligned}
$$

Next, let us look for a formal solution of the equation (3.2) in the form

$$
\psi=\sum_{k \geq 1} \psi_{k}\left(t, x, w_{0}, \ldots, w_{k-1}\right) \in \sum_{k \geq 1} \mathcal{H}_{k, R}\left[t, w_{0}, \ldots, w_{k-1}\right] .
$$

with $\left(\partial \psi_{1} / \partial w_{0}\right)=1$. As in the last part of $\S 1$, we set

$$
\begin{aligned}
& F\left(t, x, w_{0}, w_{1}\right)=a(x) t+\lambda(x) w_{0}+R_{2}\left(t, x, w_{0}, w_{1}\right), \\
& R_{2}\left(t, x, w_{0}, w_{1}\right)=\sum_{i+j+\alpha \geq 2} c_{i, j, \alpha}(x) t^{i} w_{0}^{j} w_{1}^{\alpha}, \\
& \tau_{k}^{*}=t \frac{\partial}{\partial t}+\sum_{0 \leq m \leq k-1}\left(\sum_{0 \leq i \leq m} \lambda_{m, i}(x) w_{i}\right) \frac{\partial}{\partial w_{m}}, \quad k \geq 1 .
\end{aligned}
$$

The diagonal part of the vector field $\tau_{k}^{*}$ is also just the one in (1.5) and so the operator $\tau_{k}^{*}-\lambda(x)$ satisfies the Poincaré condition. Our equation (3.2) is decomposed into the following recurrent formulas:

$$
\left(\tau_{1}^{*}-\lambda(x)\right) \psi_{1}=a(x) t \quad \text { in } \mathcal{H}_{1, R}\left[t, w_{0}\right]
$$

and for $k \geq 2$

$$
\begin{aligned}
\left(\tau_{k}^{*}-\lambda(x)\right) \psi_{k}= & \sum_{2 \leq i+j+\alpha \leq k} c_{i, j, \alpha}(x) t^{i} \sum_{|p(j)|+|q(\alpha)|=k-i} \prod_{n=1}^{j} \psi_{p_{n}} \prod_{m=1}^{\alpha} D\left[\psi_{q_{m}}\right] \\
& -\sum_{0 \leq m \leq k-K} \gamma_{2}^{(m)}(x) t^{K} \frac{\partial \psi_{k-K+1}}{\partial w_{m}}
\end{aligned}
$$

in $\mathcal{H}_{k, R}\left[t, w_{0}, \ldots, w_{k-1}\right]$, where $\psi_{j} \equiv 0$ if $j \leq 0, p(j)=\left(p_{1}, \ldots, p_{j}\right) \in \mathbf{N}^{* j}, q(\alpha)=$ $\left(q_{1}, \ldots, q_{\alpha}\right) \in \mathbf{N}^{* \alpha},|p(j)|=p_{1}+\cdots+p_{j}$ and $|q(\alpha)|=q_{1}+\cdots+q_{\alpha}$. We recall:

LEMMA 3.3 (Lemma 3.2.2 in [15]). (1) If $\lambda(x) \neq 1$ on $D_{R}$, the equation $\left(\tau_{1}^{*}-\right.$ $\lambda(x)) \psi_{1}=a(x) t$ has a solution $\psi_{1} \in \mathcal{H}_{1, R}\left[t, w_{0}\right]$ of the form

$$
\psi_{1}=\frac{a(x)}{1-\lambda(x)} t+\beta(x) w_{0}
$$

and $\beta(x) \in \mathcal{O}_{R}$ can be chosen arbitrarily. 
(2) Let $k \geq 2$. If $|i+\lambda(x)(j-1)| \neq 0$ on $D_{R}$ for any $(i, j) \in \mathbf{N} \times \mathbf{N}$ with $i+j=k$, then for any $f_{k} \in \mathcal{H}_{k, R}\left[t, w_{0}, \ldots, w_{k-1}\right]$ the equation $\left(\tau_{k}^{*}-\lambda(x)\right) \psi_{k}=f_{k}$ has a unique solution $\psi_{k} \in \mathcal{H}_{k, R}\left[t, w_{0}, \ldots, w_{k-1}\right]$.

In the case $K \geq 2$, the terms $\psi_{1}=w_{0}+(a(x) /(1-\lambda(x))) t$ and $\psi_{k}\left(t, x, u_{0}, \ldots, u_{k-1}\right)$ $(2 \leq k \leq K-1)$ are determined by Lemma 3.3, and (3.8) $K$ is written as

$$
\begin{aligned}
\left(\tau_{K}^{*}-\right. & \lambda(x)) \psi_{K} \\
& =-\gamma_{2}(x) t^{K}+\sum_{2 \leq i+j+\alpha \leq K} c_{i, j, \alpha}(x) t^{i} \sum_{|p(j)|+|q(\alpha)|=K-i} \prod_{n=1}^{j} \psi_{p_{n}} \prod_{m=1}^{\alpha} D\left[\psi_{q_{m}}\right] .
\end{aligned}
$$

If we set

$$
\psi_{K}=Y(x) t^{K}+\sum_{i+|j|=K,(i,|j|) \neq(K, 0)} \psi_{i, j}(x) t^{i} w_{0}^{j_{0}} w_{1}^{j_{1}} \cdots w_{K-1}^{j_{K-1}},
$$

by a calculation we see that $\left\{\psi_{i, j}(x) \in \mathcal{O}_{R} ; i+|j|=K,(i,|j|) \neq(K, 0)\right\}$ are also uniquely determined and they are independent of $Y(x)$ and $\gamma_{2}(x)$. If we denote by $\psi_{k, 0}(x)$ the coefficient of $t^{k}$ in $\psi_{k}\left(t, x, w_{0}, \ldots, w_{k-1}\right)(k=1,2, \ldots, K-1)$, by comparing the coefficients of $t^{K}$ in the both sides of (3.9) we have the equation

$$
\begin{aligned}
(K- & \lambda(x)) Y(x) \\
& =-\gamma_{2}(x)+\sum_{2 \leq i+j+\alpha \leq K} c_{i, j, \alpha}(x) \sum_{|p(j)|+|q(\alpha)|=K-i} \prod_{n=1}^{j} \psi_{p_{n}, 0} \prod_{m=1}^{\alpha} \frac{\partial \psi_{q_{m}, 0}}{\partial x} .
\end{aligned}
$$

Therefore, if we set

$$
\begin{aligned}
\gamma_{2}(x)= & -(K-\lambda(x)) Y(x) \\
& +\sum_{2 \leq i+j+\alpha \leq K} c_{i, j, \alpha}(x) \sum_{|p(j)|+|q(\alpha)|=K-i} \prod_{n=1}^{j} \psi_{p_{n}, 0} \prod_{m=1}^{\alpha} \frac{\partial \psi_{q_{m}, 0}}{\partial x}
\end{aligned}
$$

we have the relation (3.10); this yields (3.9) (that is, $(3.8)_{K}$ ).

For $k \geq K+1$, the situation is the same as in the non-resonant case, and so by Lemma 3.3 we can uniquely determine $\psi_{k}\left(t, x, w_{0}, \ldots, w_{k-1}\right) \in \mathcal{H}_{k, R}\left[t, w_{0}, \ldots, w_{k-1}\right]$ inductively on $k$ so that $(3.8)_{k}$ is satisfied for $k \geq K+1$. This proves the part (2), and we have

(3.11) $\mu_{2}(x)=\sum_{2 \leq i+j+\alpha \leq K} c_{i, j, \alpha}(x)\left[\sum_{|p(j)|+|q(\alpha)|=K-i} \psi_{p_{1}, 0} \cdots \psi_{p_{j}, 0} \frac{\partial \psi_{q_{1}, 0}}{\partial x} \cdots \frac{\partial \psi_{q_{\alpha}, 0}}{\partial x}\right]$.

Moreover, we have 
Proposition 3.4. Let

$$
\psi=\sum_{k \geq 1} \psi_{k}\left(t, x, w_{0}, \ldots, w_{k-1}\right) \in \sum_{k \geq 1} \mathcal{H}_{k, R}\left[t, w_{0}, \ldots, w_{k-1}\right]
$$

be the formal solution of (3.2) constructed in (2) of Proposition 3.1. Then the term $\psi_{k}$ (for $k \geq 2$ ) has the form

$$
\psi_{k}=\sum_{(i, j) \in J_{k}} \phi_{i, j}(x) t^{i} w_{0}^{j_{0}} w_{1}^{j_{1}} \cdots w_{k-1}^{j_{k-1}},
$$

where $j=\left(j_{0}, j_{1}, \ldots, j_{k-1}\right)$ and

$$
J_{k}=\left\{(i, j) \in \mathbf{N} \times \mathbf{N}^{k} ; i+|j|=k,\langle j\rangle \leq i+j_{0}+2\left(\left|j^{\prime}\right|-1\right)\right\}
$$

with the notations $|j|=j_{0}+j_{1}+\cdots+j_{k-1},\langle j\rangle=j_{1}+2 j_{2}+\cdots+(k-1) j_{k-1}$ and $\left|j^{\prime}\right|=j_{1}+j_{2}+\cdots+j_{k-1}$.

PROOF. Set $\mathcal{G}_{1}=\mathcal{H}_{1, R}\left[t, w_{0}\right]$, and denote by $\mathcal{G}_{k}$ (with $k \geq 2$ ) the set of all functions $f_{k}\left(t, x, w_{0}, \ldots, w_{k-1}\right) \in \mathcal{H}_{k, R}\left[t, w_{0}, \ldots, w_{k-1}\right]$ of the form

$$
f_{k}=\sum_{(i, j) \in J_{k}} f_{i, j}(x) t^{i} w_{0}^{j_{0}} w_{1}^{j_{1}} \cdots w_{k-1} j^{j_{k-1}} .
$$

Then we have the following properties:

1) $t(\partial / \partial t) \mathcal{G}_{k} \subset \mathcal{G}_{k}$ for any $k \in \mathbf{N}^{*}$,

2) $t\left(\partial / \partial w_{m}\right) \mathcal{G}_{k} \subset \mathcal{G}_{k}$ for any $m \in \mathbf{N}$ and $k \in \mathbf{N}^{*}$,

3) $w_{i}\left(\partial / \partial w_{m}\right) \mathcal{G}_{k} \subset \mathcal{G}_{k}$ for any $i, m \in \mathbf{N}$ with $0 \leq i \leq m$ and $k \in \mathbf{N}^{*}$,

4) $\mathcal{G}_{k} \times \mathcal{G}_{l} \subset \mathcal{G}_{k+l}$ for any $k, l \in \mathbf{N}^{*}$,

5) $w_{m+1}\left(\partial / \partial w_{m}\right) \mathcal{G}_{k} \times \mathcal{G}_{l} \subset \mathcal{G}_{k+l}$ for any $m \in \mathbf{N}$ and $k, l \in \mathbf{N}^{*}$,

6) $w_{m+1}\left(\partial / \partial w_{m}\right) \mathcal{G}_{k} \times w_{p+1}\left(\partial / \partial w_{p}\right) \mathcal{G}_{l} \subset \mathcal{G}_{k+l}$ for any $m, p \in \mathbf{N}$ and $k, l \in \mathbf{N}^{*}$.

The properties 5) and 6) imply that $D\left[\mathcal{G}_{k}\right] \times \mathcal{G}_{l} \subset \mathcal{G}_{k+l}$ and $D\left[\mathcal{G}_{k}\right] \times D\left[\mathcal{G}_{l}\right] \subset \mathcal{G}_{k+l}$ hold for any $k, l \in \mathbf{N}^{*}$. By combining the properties 1)-6) with the construction of the formal solution we can obtain the result.

3.2. Formal solutions of $(\Phi)$ and $(\Psi)$. In Proposition 3.1 we have constructed formal solutions $\phi\left(t, x, u_{0}, u_{1}, \ldots\right)$ and $\psi\left(t, x, w_{0}, w_{1}, \ldots\right)$ of (3.1) and (3.2) for some $\gamma_{1}(x)$ and $\gamma_{2}(x)$, respectively. If $\gamma_{1}(x)=\gamma_{2}(x)$ holds, we can take $\gamma(x)\left(=\gamma_{1}(x)=\gamma_{2}(x)\right)$ so that the equation

$$
t \frac{\partial w}{\partial t}=\lambda(x) w+\gamma(x) t^{K}
$$

is a candidate of the normal form of our Briot-Bouquet type partial differential equation (1.1), and we can regard $\phi\left(t, x, u_{0}, u_{1}, \ldots\right)$ and $\psi\left(t, x, w_{0}, w_{1}, \ldots\right)$ as formal solutions of $(\Phi)$ and $(\Psi)$, respectively. 
The following proposition gives a necessary and sufficient condition for this condition (that is, $\gamma_{1}(x)=\gamma_{2}(x)$ ) to be valid:

PROPOSITION 3.5. In the above situation, we can find a holomorphic function $P(x) \in$ $\mathcal{O}_{R}$ which satisfies the following properties:

(1) The following three conditions are equivalent:

$$
\begin{array}{ll}
(1-\mathrm{i}) & u_{0}=\psi\left(t, x, \phi, D[\phi], D^{2}[\phi], \ldots\right) \text { holds in } \mathcal{M}_{R}[t, \vec{u}] \\
(1-\mathrm{ii}) & w_{0}=\phi\left(t, x, \psi, D[\psi], D^{2}[\psi], \ldots\right) \text { holds in } \mathcal{M}_{R}[t, \vec{w}] ; \\
(1-\mathrm{iii}) & X(x)+Y(x)=P(x) .
\end{array}
$$

(2) The following two conditions are equivalent:

$$
\begin{array}{ll}
(2-\mathrm{i}) & \gamma_{1}(x)=\gamma_{2}(x), \\
(2-\mathrm{ii}) & (K-\lambda(x))(X(x)+Y(x))=(K-\lambda(x)) P(x) .
\end{array}
$$

To prove this, we recall some general results in [14] and [15]:

Proposition 3.6. (1) Let $\phi\left(t, x, u_{0}, u_{1}, u_{2}, \ldots\right)$ be of the form (3.3), and suppose that $\left(\partial \phi_{1} / \partial u_{0}\right) \neq 0$ holds on $D_{R}$. Then the equation

$$
w_{0}=\phi\left(t, x, \psi, D[\psi], D^{2}[\psi], \ldots\right) \quad \text { in } \sum_{k \geq 1} \mathcal{H}_{k, R}\left[t, w_{0}, \ldots, w_{k-1}\right]
$$

has a unique formal solution $\psi\left(t, x, w_{0}, w_{1}, w_{2}, \ldots\right)$ of the form (3.7) satisfying $\left(\partial \psi_{1} / \partial w_{0}\right) \neq 0$ on $D_{R}$, and it satisfies also

$$
u_{0}=\psi\left(t, x, \phi, D[\phi], D^{2}[\phi], \ldots\right) \text { in } \sum_{k \geq 1} \mathcal{H}_{k, R}\left[t, u_{0}, \ldots, u_{k-1}\right] .
$$

(2) Moreover, if $\phi\left(t, x, u_{0}, u_{1}, \ldots\right)$ is a formal solution of the equation

$$
t \frac{\partial \phi}{\partial t}+\sum_{m \geq 0} D^{m}[F]\left(t, x, u_{0}, \ldots, u_{m+1}\right) \frac{\partial \phi}{\partial u_{m}}=G(t, x, \phi, D[\phi]),
$$

for some holomorphic functions $F(t, x, u, v)$ and $G(t, x, u, v)$ in a neighborhood of $(0,0,0,0) \in \mathbf{C}_{t} \times \mathbf{C}_{x} \times \mathbf{C}_{u} \times \mathbf{C}_{v}$, then the unique solution $\psi\left(t, x, w_{0}, w_{1}, \ldots\right)$ of (3.14) in (1) is a formal solution of the reverse equation

$$
t \frac{\partial \psi}{\partial t}+\sum_{m \geq 0} D^{m}[G]\left(t, x, w_{0}, \ldots, w_{m+1}\right) \frac{\partial \psi}{\partial w_{m}}=F(t, x, \psi, D[\psi]) .
$$

Proposition 3.7. Let $F(t, x, u, v), G(t, x, u, v)$ and $H(t, x, u, v)$ be holomorphic functions in a neighborhood of $(0,0,0,0) \in \mathbf{C}_{t} \times \mathbf{C}_{x} \times \mathbf{C}_{u} \times \mathbf{C}_{v}$. Let $\phi\left(t, x, u_{0}, u_{1}, \ldots\right)$ be a formal solution of

$$
t \frac{\partial \phi}{\partial t}+\sum_{m \geq 0} D^{m}[F]\left(t, x, u_{0}, \ldots, u_{m+1}\right) \frac{\partial \phi}{\partial u_{m}}=G(t, x, \phi, D[\phi])
$$


and let $z\left(t, x, w_{0}, w_{1}, \ldots\right)$ be a formal solution of

$$
t \frac{\partial z}{\partial t}+\sum_{m \geq 0} D^{m}[G]\left(t, x, w_{0}, \ldots, w_{m+1}\right) \frac{\partial z}{\partial w_{m}}=H(t, x, z, D[z]) .
$$

Then, the composition $\zeta\left(t, x, u_{0}, u_{1}, u_{2}, \ldots\right)=z\left(t, x, \phi, D[\phi], D^{2}[\phi], \ldots\right)$ is a formal solution of

$$
t \frac{\partial \zeta}{\partial t}+\sum_{m \geq 0} D^{m}[F]\left(t, x, u_{0}, \ldots, u_{m+1}\right) \frac{\partial \zeta}{\partial u_{m}}=H(t, x, \zeta, D[\zeta]) .
$$

The part (1) of Proposition 3.6 is proved in [Lemma 2.9 in [15]], and the part (2) follows from [Propositions 2.4 and 2.8 in [15]]. Since the composition of two couplings is also a coupling, by [Proposition 3.4.1 in [14]] we have the result in Proposition 3.7.

By using these results, let us give a proof of Proposition 3.5.

Proof of Proposition 3.5 In the CASE $K \geq 2$. Take any $X(x) \in \mathcal{O}_{R}$ and $Y(x) \in$ $\mathcal{O}_{R}$. Let

$$
\begin{aligned}
& \phi=\sum_{k \geq 1} \phi_{k}\left(t, x, u_{0}, \ldots, u_{k-1}\right)=u_{0}+\frac{-a(x)}{1-\lambda(x)} t+\cdots+X(x) t^{K}+\cdots, \\
& \psi=\sum_{k \geq 1} \psi_{k}\left(t, x, w_{0}, \ldots, w_{k-1}\right)=w_{0}+\frac{a(x)}{1-\lambda(x)} t+\cdots+Y(x) t^{K}+\cdots
\end{aligned}
$$

be the formal solutions constructed in (1) and (2) of Proposition 3.1, respectively.

First, let us show that (1-i) implies (1-ii). Suppose the condition (1-i). By (1) of Proposition 3.6 we see that the equation

$$
u_{0}=\psi\left(t, x, \eta, D[\eta], D^{2}[\eta], \ldots\right) \quad \text { in } \sum_{k \geq 1} \mathcal{H}_{k, R}\left[t, u_{0}, \ldots, u_{k-1}\right]
$$

has a unique formal solution $\eta\left(t, x, u_{0}, u_{1}, \ldots\right) \in \sum_{k \geq 1} \mathcal{H}_{k, R}\left[t, u_{0}, \ldots, u_{k-1}\right]$, and it satisfies

$$
w_{0}=\eta\left(t, x, \psi, D[\psi], D^{2}[\psi], \ldots\right) \quad \text { in } \sum_{k \geq 1} \mathcal{H}_{k, R}\left[t, w_{0}, \ldots, w_{k-1}\right]
$$

Since (1-i) is supposed, $\phi\left(t, x, u_{0}, u_{1}, \ldots\right)$ is also a formal solution of (3.20), and by the uniqueness of the solution we have $\eta\left(t, x, u_{0}, u_{1}, \ldots\right)=\phi\left(t, x, u_{0}, u_{1}, \ldots\right)$. Thus, by (3.21) we have the condition (1-ii).

The proof of the converse is the same as above, and so we have the equivalence of (1-i) and (1-ii).

Next, let us show that (1-ii) implies (1-iii) for some holomorphic function $P(x) \in \mathcal{O}_{R}$. 
Suppose the condition (1-ii); by substituting (3.18) and (3.19) into the relation (1-ii) we have

$$
0=\sum_{k \geq 2} \psi_{k}+\sum_{k \geq 2} \phi_{k}\left(t, x, \sum_{p \geq 1} \psi_{p}, \ldots, \sum_{p \geq 1} D^{k-1}\left[\psi_{p}\right]\right)
$$

(with $\left.\psi_{1}=w_{0}+(a(x) /(1-\lambda(x))) t\right)$. Therefore, by looking at only the homogeneous part of degree $K$ in $\left(t, w_{0}, \ldots, w_{K-1}\right)$ (that is, the part belonging in the class $\left.\mathcal{H}_{K, R}\left[t, w_{0}, \ldots, w_{K-1}\right]\right)$ we have

$$
0=\psi_{K}+\sum_{2 \leq k \leq K}\left[\sum_{\begin{array}{c}
l+|\alpha|=k \\
l+|p(\alpha)|=K
\end{array}} \phi_{l, \alpha} t^{l} \prod_{h=0}^{k-1}\left(D^{h}\left[\psi_{p_{h}(1)}\right] \times \cdots \times D^{h}\left[\psi_{p_{h}\left(\alpha_{h}\right)}\right]\right)\right]
$$

where $\psi_{K}=\psi_{K}\left(t, x, w_{0}, \ldots, w_{K-1}\right), \alpha=\left(\alpha_{0}, \ldots, \alpha_{k-1}\right),|\alpha|=\alpha_{0}+\cdots+\alpha_{k-1}$, $p(\alpha)=\left(p_{h}(i) ; 0 \leq h \leq k-1,1 \leq i \leq \alpha_{h}\right) \in \mathbf{N}^{*|\alpha|},|p(\alpha)|=\sum_{0 \leq h \leq k-1}\left(p_{h}(1)+\right.$ $\left.\cdots+p_{h}\left(\alpha_{h}\right)\right), \phi_{l, \alpha}=\phi_{l, \alpha}(x)$ is the coefficient of $t^{l} u^{\alpha}$ in $\phi_{k}\left(t, x, u_{0}, \ldots, u_{k-1}\right)$, and $\psi_{p_{h}(q)}=\psi_{p_{h}(q)}\left(t, x, w_{0}, \ldots, w_{p_{h}(q)-1}\right)\left(1 \leq q \leq \alpha_{h}\right)$. Thus, by looking at the coefficients of $t^{K}$ we have the relation

$$
0=Y(x)+X(x)+\sum_{2 \leq k \leq K}\left[\sum_{\substack{l+|\alpha|=k \\ l+|p(\alpha)|=K, l<K}} \phi_{l, \alpha} \prod_{h=0}^{k-1}\left(\psi_{p_{h}(1), 0}^{(h)} \times \cdots \times \psi_{p_{h}\left(\alpha_{h}\right), 0}^{(h)}\right)\right]
$$

where $\psi_{p, 0}=\psi_{p, 0}(x)$ is the coefficient of $t^{p}$ in $\psi_{p}\left(t, x, w_{0}, \ldots, w_{p-1}\right)$ and $\psi_{p, 0}^{(h)}=$ $(\partial / \partial x)^{h} \psi_{p, 0}(x)$. Thus, by setting

$$
P(x)=-\sum_{2 \leq k \leq K}\left[\sum_{\substack{l+|\alpha|=k \\ l+|p(\alpha)|=K, l<K}} \phi_{l, \alpha} \prod_{h=0}^{k-1}\left(\psi_{p_{h}(1), 0}^{(h)} \times \cdots \times \psi_{p_{h}\left(\alpha_{h}\right), 0}^{(h)}\right)\right]
$$

we have the relation $X(x)+Y(x)=P(x)$ on $D_{R}$. Since the functions $\psi_{p, 0}(2 \leq p \leq K-1)$, $\phi_{k}(2 \leq k \leq K-1)$ and $\left\{\phi_{l, \alpha} ; l+|\alpha|=K,(l,|\alpha|) \neq(K, 0)\right\}$ are determined only by $F(t, x, u, v)$ we can say that the function $P(x)$ is also determined only by $F(t, x, u, v)$. This proves that (1-ii) implies (1-iii).

Conversely, let $P(x)$ be as above, and let us show that (1-iii) implies (1-ii). Suppose the condition (1-iii); this means that the coefficient of $t^{K}$ of the formal series $\zeta\left(t, x, w_{0}, w_{1}, \ldots\right)=\phi\left(t, x, \psi, D[\psi], D^{2}[\psi], \ldots\right)$ is equal to 0 on $D_{R}$ and so we have

$$
\begin{aligned}
\zeta\left(t, x, w_{0}, w_{1}, \ldots\right) & =w_{0}+\cdots+0 \times t^{K}+\cdots \\
& =\sum_{k \geq 1} \zeta_{k}\left(t, x, w_{0}, \ldots, w_{k-1}\right) \in \sum_{k \geq 1} \mathcal{H}_{k, R}\left[t, w_{0}, \ldots, w_{k-1}\right]
\end{aligned}
$$


where $\zeta_{1}=w_{0}$ and $\zeta_{K}\left(t, x, w_{0}, \ldots, w_{K-1}\right)$ has the form

$$
\zeta_{K}=\sum_{i+|j|=K,(i,|j|) \neq(K, 0)} \zeta_{i, j}(x) t^{i} w_{0}^{j_{0}} w_{1}^{j_{1}} \cdots w_{K-1}^{j_{K-1}} ;
$$

moreover, by Proposition 3.7 we have the relation

$$
t \frac{\partial \zeta}{\partial t}+\sum_{m \geq 0}\left(\gamma_{2}^{(m)}(x) t^{K}+\sum_{0 \leq i \leq m} \lambda_{m, i}(x) w_{i}\right) \frac{\partial \zeta}{\partial w_{m}}=\lambda(x) \zeta+\gamma_{1}(x) t^{K} .
$$

Since $K \geq 2$, by (3.23) we have

$$
\left(\tau_{k}^{*}-\lambda(x)\right) \zeta_{k}=0, \quad 2 \leq k \leq K-1
$$

(where $\tau_{k}^{*}$ is the same as in $(3.8)_{k}$ ), and so we have $\zeta_{k} \equiv 0$ for $2 \leq k \leq K-1$. If $k=K$, by the condition $\zeta_{1}=w_{0}$ and (3.23) we have

$$
\left(\tau_{K}^{*}-\lambda(x)\right) \zeta_{K}=\gamma_{1}(x) t^{K}-\gamma_{2}(x) t^{K} ;
$$

since the coefficient of $t^{K}$ of $\zeta_{K}\left(t, x, w_{0}, \ldots, w_{K-1}\right)$ is equal to 0 , we have $\zeta_{K} \equiv 0$ and $\gamma_{1}(x)=\gamma_{2}(x)$ (which is (2-i)). If $k \geq K+1$, by induction on $k$, we can see that $\zeta_{k} \equiv 0$ holds for all $k \geq K+1$ in the same way as above.

Thus, we have shown that $\zeta_{1}=w_{0}$ and $\zeta_{k} \equiv 0$ for all $k \geq 2$; this implies that $\zeta\left(t, x, w_{0}, w_{1}, \ldots\right)=w_{0}$. Therefore, by the definition of $\zeta\left(t, x, w_{0}, w_{1}, \ldots\right)$ we have $w_{0}=\phi\left(t, x, \psi, D[\psi], D^{2}[\psi], \ldots\right)$. This proves (1-ii).

Summing up all the above results, we have proved the part (1) of Proposition 3.5. At the same time, we have shown that the condition $X(x)+Y(x)=P(x)$ implies the condition $\gamma_{1}(x)=\gamma_{2}(x)$.

Now, let us show that (2-i) implies (2-ii). Suppose the condition (2-i). Let us consider the equation

$$
w_{0}=\phi\left(t, x, \xi, D[\xi], D^{2}[\xi], \ldots\right) \quad \text { in } \sum_{k \geq 1} \mathcal{H}_{k, R}\left[t, w_{0}, \ldots, w_{k-1}\right]
$$

By (1) of Proposition 3.6 we see that (3.24) has a unique formal solution of the form

$$
\xi=w_{0}+\frac{a(x)}{1-\lambda(x)} t+\sum_{k \geq 2} \xi_{k}\left(t, x, w_{0}, \ldots, w_{k-1}\right) \in \sum_{k \geq 1} \mathcal{H}_{k, R}\left[t, w_{0}, \ldots, w_{k-1}\right] ;
$$

we set $\xi_{1}=w_{0}+(a(x) /(1-\lambda(x))) t$ and

$$
\xi_{K}=Y_{\xi}(x) t^{K}+\sum_{i+|j|=K,(i,|j|) \neq(K, 0)} \xi_{i, j}(x) t^{i} w_{0}^{j_{0}} w_{1}^{j_{1}} \cdots w_{K-1}^{j_{K-1}} .
$$

Since $\phi\left(t, x, u_{0}, u_{1}, \ldots\right)$ is a formal solution of (3.1) with $\left(\partial \phi / \partial u_{0}\right)(0, x, 0,0, \ldots)=1$, by 
(2) of Proposition 3.6 we have

$$
t \frac{\partial \xi}{\partial t}+\sum_{m \geq 0}\left(\gamma_{1}^{(m)}(x) t^{K}+\sum_{0 \leq i \leq m} \lambda_{m, i}(x) w_{i}\right) \frac{\partial \xi}{\partial w_{m}}=F(t, x, \xi, D[\xi]) .
$$

By solving this equation and by comparing $\xi_{k}$ with $\psi_{k}$ in (3.19) we see that $\xi_{k}\left(t, x, w_{0}, \ldots, w_{k-1}\right)=\psi_{k}\left(t, x, w_{0}, \ldots, w_{k-1}\right)($ for $2 \leq k \leq K-1)$ and $\xi_{i, j}(x)=\psi_{i, j}(x)$ (for $i+|j|=K$ with $(i,|j|) \neq(K, 0))$.

Since (3.24) holds, we can apply the conclusion of the part (1), and obtain the relation $X(x)+Y_{\xi}(x)=P_{\xi}(x)$ for the holomorphic function $P_{\xi}(x) \in \mathcal{O}_{R}$ given by

$$
P_{\xi}(x)=-\sum_{2 \leq k \leq K}\left[\sum_{\substack{l+|\alpha|=k \\ l+|p(\alpha)|=K, l<K}} \phi_{l, \alpha} \prod_{h=0}^{k-1}\left(\xi_{p_{h}(1), 0}^{(h)} \times \cdots \times \xi_{p_{h}\left(\alpha_{h}\right), 0}^{(h)}\right)\right] .
$$

Since $\xi_{p, 0}(x)=\psi_{p, 0}(x)$ for all $1 \leq p \leq K-1$, by (3.22) and (3.26) we have $P(x)=P_{\xi}(x)$ and so we have the relation $X(x)+Y_{\xi}(x)=P(x)$.

Here, we recall that in Proposition 3.1 the function $\gamma_{2}(x)$ is expressed as $\gamma_{2}(x)=$ $-Y(x)(K-\lambda(x))+\mu_{2}(x)$ and $\mu_{2}(x)$ is calculated by the formula (3.11). Similarly, by applying the same argument to (3.25) we have $\gamma_{1}(x)=-Y_{\xi}(x)(K-\lambda(x))+\mu_{2, \xi}(x)$ and $\mu_{2, \xi}(x)$ is calculated by

$$
\mu_{2, \xi}(x)=\sum_{2 \leq i+j+\alpha \leq K} c_{i, j, \alpha}(x)\left[\sum_{|p(j)|+|q(\alpha)|=K-i} \xi_{p_{1}, 0} \cdots \xi_{p_{j}, 0} \frac{\partial \xi_{q_{1}, 0}}{\partial x} \cdots \frac{\partial \xi_{q_{\alpha}, 0}}{\partial x}\right] .
$$

Since $\xi_{p, 0}(x)=\psi_{p, 0}$ holds for $1 \leq p \leq K-1$, we have $\mu_{2}(x)=\mu_{2, \xi}(x)$. Since $\gamma_{1}(x)=$ $\gamma_{2}(x)$ is supposed in (2-i), by the two relations $\gamma_{2}(x)=-Y(x)(K-\lambda(x))+\mu_{2}(x)$ and $\gamma_{1}(x)=-Y_{\xi}(x)(K-\lambda(x))+\mu_{2, \xi}(x)$ we have $Y(x)(K-\lambda(x))=Y_{\xi}(x)(K-\lambda(x))$ on $D_{R}$. Thus, by combining this with the known relation $X(x)+Y_{\xi}(x)=P(x)$ we obtain the equality

$$
P(x)(K-\lambda(x))=\left(X(x)+Y_{\xi}(x)\right)(K-\lambda(x))=(X(x)+Y(x))(K-\lambda(x)) ;
$$

this proves (2-ii).

Lastly, let us show that (2-ii) implies (2-i). Suppose the condition (2-ii), that is, $(K-$ $\lambda(x))(X(x)+Y(x))=(K-\lambda(x)) P(x)$. If $\lambda(x) \not \equiv K$, we have $X(x)+Y(x)=P(x)$ and so, as is already proved, we have the condition $\gamma_{1}(x)=\gamma_{2}(x)$ on $D_{R}$. If $\lambda(x) \equiv K$ on $D_{R}$, we have $\gamma_{1}(x)=X(x)(K-\lambda(x))+\mu_{1}(x)=\mu_{1}(x)$ and $\gamma_{2}(x)=-Y(x)(K-\lambda(x))+\mu_{2}(x)=$ $\mu_{2}(x)$; this means that $\gamma_{1}(x)$ and $\gamma_{2}(x)$ are independent of the choice of $X(x)$ and $Y(x)$. In this case, by taking other $X^{0}(x)$ and $Y^{0}(x)$ with $X^{0}(x)+Y^{0}(x)=P(x)$ and by applying the same argument as above we have the result $\gamma_{1}(x)=\gamma_{2}(x)$. This proves (2-i).

Thus, we have proved the part (2) of Proposition 3.5. 
Proof Of Proposition 3.5 IN THE CASE $K=1$. Let

$$
\begin{aligned}
& \phi=u_{0}+X(x) t+\sum_{k \geq 2} \phi_{k}\left(t, x, u_{0}, \ldots, u_{k-1}\right), \\
& \psi=w_{0}+Y(x) t+\sum_{k \geq 2} \psi_{k}\left(t, x, w_{0}, \ldots, w_{k-1}\right)
\end{aligned}
$$

be the formal solutions constructed in (1) and (2) of Proposition 3.1, respectively. The equivalence of (1-i) and (1-ii) is verified in the same way as above. If we suppose the condition (1-ii), by looking at the coefficient of $t$ in the formula (1-ii) we have $X(x)+Y(x)=0$, and so if we set $P(x)=0$ we have the condition (1-iii). Conversely, if $X(x)+Y(x)=0$ holds, by calculating the formal solution of (3.23) we have the condition (1-ii). This proves the part (1) of Proposition 3.5.

Since we know $\gamma_{1}(x)=(1-\lambda(x)) X(x)+a(x)$ and $\gamma_{2}(x)=-(1-\lambda(x)) Y(x)+a(x)$, by the condition $P(x)=0$ we have the part (2).

Corollary 3.8. Let $\mu_{1}(x), \mu_{2}(x)$ and $P(x)$ be the functions in Propositions 3.1 and 3.5. Then, these are determined only by $F(t, x, u, v)$ and they satisfy the relation

$$
\mu_{2}(x)-\mu_{1}(x)=(K-\lambda(x)) P(x) \text { on } D_{R} .
$$

Proof. Take any $X(x)$ and $Y(x)$ such that $X(x)+Y(x)=P(x)$; then we have $\gamma_{1}(x)=\gamma_{2}(x)$ and so $X(x)(K-\lambda(x))+\mu_{1}(x)=-Y(x)(K-\lambda(x))+\mu_{2}(x)$. Therefore, we have $\mu_{2}(x)-\mu_{1}(x)=(K-\lambda(x))(X(x)+Y(x))$. Since $X(x)+Y(x)=P(x)$ holds, we have the equality $\mu_{2}(x)-\mu_{1}(x)=(K-\lambda(x)) P(x)$.

Thus, by taking $X(x)$ and $Y(x)$ so that $X(x)+Y(x)=P(x)$ and by setting $\gamma(x)=$ $\gamma_{1}(x)=\gamma_{2}(x)$ we have the following result.

Proposition 3.9. Suppose the conditions $\left.\left.\left.\mathrm{A}_{1}\right), \mathrm{A}_{2}\right), \mathrm{A}_{3}\right)$ and $\lambda(0)=K \in$ $\{1,2,3, \ldots\}$. Let $R>0$ be sufficiently small. Then, we have a holomorphic function $\gamma(x) \in \mathcal{O}_{R}$ which satisfies the following:

(1) The equation $(\Phi)$ has a formal solution

$$
\phi=u_{0}-\alpha(x) t+\sum_{k \geq 2} \phi_{k}\left(t, x, u_{0}, \ldots, u_{k-1}\right)
$$

with $\alpha(x) \in \mathcal{O}_{R}$ and $\phi_{k}\left(t, x, u_{0}, \ldots, u_{k-1}\right) \in \mathcal{H}_{k, R}\left[t, u_{0}, \ldots, u_{k-1}\right]($ for $k \geq 2)$.

(2) The equation $(\Psi)$ has a formal solution

$$
\psi=w_{0}+\alpha(x) t+\sum_{k \geq 2} \psi_{k}\left(t, x, w_{0}, \ldots, w_{k-1}\right)
$$

with $\psi_{k}\left(t, x, w_{0}, \ldots, w_{k-1}\right) \in \mathcal{H}_{k, R}\left[t, w_{0}, \ldots, w_{k-1}\right]($ for $k \geq 2)$, and $\psi_{k}$ (for $\left.k \geq 2\right)$ has the form (3.12) (in Proposition 3.4).

(3) Moreover, we have the following two identities: $u_{0}=\psi\left(t, x, \phi, D[\phi], D^{2}[\phi], \ldots\right)$ in $\mathcal{M}_{R}[t, \vec{u}]$, and $w_{0}=\phi\left(t, x, \psi, D[\psi], D^{2}[\psi], \ldots\right)$ in $\mathcal{M}_{R}[t, \vec{w}]$. 
By Proposition 2.1, we see that if $u(t, x)$ is a holomorphic solution of

$$
t \frac{\partial u}{\partial t}=F\left(t, x, u, \frac{\partial u}{\partial x}\right)
$$

and if $w(t, x)=\phi(t, x, u, \partial u / \partial x, \ldots)$ (we write this as $w=\Phi[u]$ ) is well-defined, then the function $w(t, x)$ is a holomorphic solution of

$$
t \frac{\partial w}{\partial t}=\lambda(x) w+\gamma(x) t^{K} .
$$

Similarly, if $w(t, x)$ is a holomorphic solution of (3.33) and if $u(t, x)=\psi(t, x$, $w, \partial w / \partial x, \ldots$ ) (we write this as $u=\Phi[w]$ ) is well-defined, then the function $u(t, x)$ is a holomorphic solution of (3.32). Moreover, by the result (3) of Proposition 3.9 we have $u=\Psi[\Phi[u]]$ and $w=\Phi[\Psi[w]]$. Therefore, by Proposition 3.9 and Definition 2.5 we can conclude that two equations (3.32) and (3.33) are formally equivalent.

Hence, by setting $\mu(x)=\mu_{2}(x), \gamma(x)=-Y(x)(K-\lambda(x))+\mu(x)$ and $X(x)=$ $P(x)-Y(x)$ (for any $Y(x))$, we have

THEOREM 3.10. Suppose the conditions $\left.\left.\left.\mathrm{A}_{1}\right), \mathrm{A}_{2}\right), \mathrm{A}_{3}\right)$ and $\lambda(0)=K \in\{1,2, \ldots\}$. Then, there is a holomorphic function $\mu(x) \in \mathcal{O}_{x}$ such that for any $\gamma(x) \in\left(\mathcal{O}_{x}(K-\lambda(x))+\right.$ $\mu(x))$ the following two equations are formally equivalent:

$$
t \frac{\partial u}{\partial t}=F\left(t, x, u, \frac{\partial u}{\partial x}\right) \text { and } t \frac{\partial w}{\partial t}=\lambda(x) w+\gamma(x) t^{K} .
$$

In the above, $\mathcal{O}_{x}$ denotes the set of all holomorphic functions $f(x)$ in a neighborhood of $x=0 \in \mathbf{C}$, and $\left(\mathcal{O}_{x}(K-\lambda(x))+\mu(x)\right)$ denotes the set of all holomorphic functions $\gamma(x)$ which is expressed in the form $\gamma(x)=f(x)(K-\lambda(x))+\mu(x)$ for some $f(x) \in \mathcal{O}_{x}$.

\section{Estimates of the formal solutions}

In this section, we take any $\gamma(x) \in\left(\mathcal{O}_{x}(K-\lambda(x))+\mu(x)\right)$ and fix it; then the coupling equations $(\Phi)$ and $(\Psi)$ are also fixed. We let

$$
\begin{aligned}
\phi & =\sum_{k \geq 1} \phi_{k}\left(t, x, u_{0}, \ldots, u_{k-1}\right) \in \sum_{k \geq 1} \mathcal{H}_{k, R}\left[t, u_{0}, \ldots, u_{k-1}\right], \\
\psi & =\sum_{k \geq 1} \psi_{k}\left(t, x, w_{0}, \ldots, w_{k-1}\right) \in \sum_{k \geq 1} \mathcal{H}_{k, R}\left[t, w_{0}, \ldots, w_{k-1}\right]
\end{aligned}
$$

be the formal solutions of $(\Phi)$ and $(\Psi)$, respectively, in Proposition 3.9. We may suppose that $\phi_{1}=u_{0}-\alpha(x) t$ and $\psi_{1}=w_{0}+\alpha(x) t$ for some $\alpha(x) \in \mathcal{O}_{R}$.

4.1. Estimates of $\psi\left(t, x, w_{0}, w_{1}, \ldots\right)$. For $s>0$ and a function $\varphi(x)$ we define the norm $\|\varphi\|_{s}$ of $\varphi(x)$ by $\|\varphi\|_{s}=\max _{|x| \leq s}|\varphi(x)|$. For $r>0, c>0, s>0$ and

$$
f_{k}=\sum_{i+|j|=k} f_{i, j}(x) t^{i} w_{0}^{j_{0}} w_{1}^{j_{1}} \cdots w_{k-1}^{j_{k-1}} \in \mathcal{H}_{k, R}\left[t, w_{0}, w_{1}, \ldots, w_{k-1}\right]
$$


we define the norm $\left\|f_{k}\right\|_{r, c, s}$ of $f_{k}$ by

$$
\left\|f_{k}\right\|_{r, c, s}=\sum_{i+|j|=k}\left\|f_{i, j}\right\|_{s} r^{i}\left(\frac{0 !}{c^{0}}\right)^{j_{0}}\left(\frac{1 !}{c^{1}}\right)^{j_{1}}\left(\frac{2 !}{c^{2}}\right)^{j_{2}} \cdots\left(\frac{(k-1) !}{c^{k-1}}\right)^{j_{k-1}} .
$$

We denote by $\mathbf{C}\{z\}$ the ring of convergent power series in $z$ with complex coefficients. We have

Proposition 4.1. Suppose the condition $\lambda(0)=K \in\{1,2, \ldots\}$; then we can find a constant $c_{0}>0$ such that the formal solution (4.2) satisfies

$$
\sum_{k \geq 1}\left\|\psi_{k}\right\|_{r, c, s} z^{k} \in \mathbf{C}\{z\}
$$

for any $r>0,0<c \leq c_{0}$ and $0<s<R$.

Proof. The proof in the case $K=1$ is the same as that of [Theorem 3.3.1 in [15]]. In the case $K \geq 2$, the proof is also quite similar to the proof of [Theorem 3.3.1 in [15]], and so we will give here only a sketch of the proof.

We note that $\gamma(x)$ is a holomorphic function in a neighborhood of $D_{R}$ and so we have $\left|\gamma^{(m)}(x)\right| \leq m ! B / \eta^{m}$ on $D_{R}(m=0,1,2, \ldots)$ for some $B>0$ and $\eta>0$. By the condition (1.4) and by the same argument as in [Lemma 3.3.2 in [15]] we can show:

LEMMA 4.2. We can find a constant $c_{0}>0$ with $0<c_{0}<\eta$ such that for $k \geq 2$, $r>0,0<c \leq c_{0}$ and $0<s \leq R$ we have the following results.

(1) If $k \geq 2$ and $k \neq K$, the term $\psi_{k}\left(t, x, w_{0}, \ldots, w_{k-1}\right) \in \mathcal{H}_{k, R}\left[t, w_{0}, \ldots, w_{k-1}\right]$ in the formal solution (4.2) satisfies

$$
\sigma k\left\|\psi_{k}\right\|_{r, c, s} \leq\left\|\left(\tau_{k}^{*}-\lambda(x)\right) \psi_{k}\right\|_{r, c, s}
$$

where $\sigma=\sigma_{0} / 2$ and $\sigma_{0}$ is the constant in (1.4).

(2) Let $\psi_{K}=\psi_{K}\left(t, x, w_{0}, \ldots, w_{K-1}\right)$ be the homogeneous part of degree $K$ of the formal solution (4.2), and let $\psi_{K, 0}(x)$ be the coefficient of $t^{K}$ of $\psi_{K}$ : we have

$$
\sigma K\left\|\psi_{K}\right\|_{r, c, s} \leq\left\|\left(\tau_{K}^{*}-\lambda(x)\right) \psi_{K}\right\|_{r, c, s}+\sigma K\left\|\psi_{K, 0}\right\|_{s} r^{K} .
$$

By a similar argument we have

LEMMA 4.3. For any $r>0,0<c \leq \eta, 0<s \leq R$ and $\psi_{k}\left(t, x, w_{0}, \ldots, w_{k-1}\right) \in$ $\mathcal{H}_{k, R}\left[t, w_{0}, \ldots, w_{k-1}\right]$ we have

$$
\sum_{0 \leq m \leq k-1}\left\|\gamma^{(m)}(x) t^{K}\left(\partial \psi_{k} / \partial w_{m}\right)\right\|_{r, c, s} \leq B r^{K} k\left\|\psi_{k}\right\|_{r, c, s} .
$$

Now, let us give a sketch of the proof of Proposition 4.1. Take any $r>0$ and $0<c \leq c_{0}$, and fix them. We will regard $s$ as a parameter with $0<s<R$. Then, by applying Lemmas 
4.2 and 4.3 to $(3.8)_{k}$ (with $\gamma_{2}(x)$ replaced by $\gamma(x)$ ) we have the following estimates for $k \geq 2$ :

$\left\|\psi_{k}\right\|_{r, c, s}$

$$
\leq \frac{1}{\sigma k}\left[\sum_{2 \leq i+j+\alpha \leq k} A_{i, j, \alpha} r^{i} \sum_{|p(j)|+|q(\alpha)|=k-i} \prod_{n=1}^{j}\left\|\psi_{p_{n}}\right\|_{r, c, s} \prod_{m=1}^{\alpha}\left\|D\left[\psi_{q_{m}}\right]\right\|_{r, c, s}+\mathscr{R}_{k}\right]
$$

with $\mathscr{R}_{k}=0$ for $2 \leq k \leq K-1$, and

$$
\mathscr{R}_{k}= \begin{cases}B r^{K}\left\|\psi_{1}\right\|_{r, c, s}+\sigma K\left\|\psi_{K, 0}\right\|_{s} r^{K}, & \text { for } k=K \\ B r^{K}(k-K+1)\left\|\psi_{k-K+1}\right\|_{r, c, s} & \text { for } k \geq K+1,\end{cases}
$$

where $A_{i, j, \alpha} \geq 0(i+j+\alpha \geq 2)$ are constants such that $\left\|c_{i, j, \alpha}\right\|_{R} \leq A_{i, j, \alpha}$ holds for all $i+j+\alpha \geq 2$ and that $\sum_{i+j+\alpha \geq 2} A_{i, j, \alpha} t^{i} Y^{j+\alpha}$ is convergent in a neighborhood of $(t, Y)=$ $(0,0) \in \mathbf{C} \times \mathbf{C}$. For simplicity we suppose: $0<R \leq 1$.

Let us take $A_{1}>0$ and $B_{1}>0$ so that

$$
\left\|\psi_{1}\right\|_{r, c, R} \leq A_{1}, \quad\left\|D\left[\psi_{1}\right]\right\|_{r, c, R} \leq(e+3 / c) A_{1} \quad \text { and } \quad\left\|\psi_{K, 0}\right\|_{R} \leq B_{1}
$$

and let us consider the equation

$$
\begin{array}{r}
Y=A_{1} z+\frac{1}{\sigma} \frac{1}{(R-s)}\left[\sum_{i+j+\alpha \geq 2} \frac{A_{i, j, \alpha} r^{i}}{(R-s)^{i+j+\alpha-2}} z^{i} Y^{j}((e+3 / c) Y)^{\alpha}\right. \\
\left.+\frac{B r^{K}}{(R-s)^{K-2}} z^{K-1} Y+\frac{\sigma K B_{1} r^{K}}{(R-s)^{K-2}} z^{K}\right]
\end{array}
$$

(where $\sigma=\sigma_{o} / 2, s$ is a parameter with $0<s<R$, and $B$ is the constant in Lemma 4.3). Then, the implicit function theorem tells us that (4.4) has a unique holomorphic solution $Y=\sum_{k \geq 1} Y(s) z^{k}$ in a neighborhood of $z=0$. By the same argument as in the proof of [Theorem 3.3.1 in [15]]. we can show:

$$
\left\|\psi_{k}\right\|_{r, c, s} \leq Y_{k}(s) \text { for any } 0<s<R \text { and } k=1,2, \ldots
$$

This proves Proposition 4.1.

4.2. Estimates of $\phi\left(t, x, u_{0}, u_{1}, \ldots\right)$. Let $\phi\left(t, x, u_{0}, u_{1}, \ldots\right)$ be the formal solution (4.1), and let $\phi_{K, 0}(x)$ be the coefficient of $t^{K}$ in this formal solution; we have

$$
\phi_{K, 0}(x) \ll \frac{C_{K, 0}}{(R-x)}
$$

for some $C_{K, 0} \geq 0$. We have:

Proposition 4.4. Suppose the condition $\lambda(0)=K \in\{1,2, \ldots\}$; then we can find constants $r_{0}>0$ and $0<c_{0}<R$ such that the formal solution (4.1) satisfies

$$
\sum_{k \geq 1}\left\|\phi_{k}\right\|_{r, c, s} z^{k} \in \mathbf{C}\{z\}
$$


for any $0<r \leq r_{0}, 0<c \leq c_{0}$ and $0<s<R$ satisfying $c+s=R$.

Proof. The proof in the case $K=1$ is the same as that of [Theorem 3.4.1 in [15]]. In the case $K \geq 2$, the proof is also quite similar to the proof of [Theorem 3.4.1 in [15]], and so we will give here only a sketch of the proof. We write

$$
\mathcal{H}_{k,(R)}\left[t, w_{0}, \ldots, w_{k-1}\right]=\bigcap_{0<\eta<R} \mathcal{H}_{k, \eta}\left[t, w_{0}, \ldots, w_{k-1}\right]
$$

Let $A_{i, j, \alpha} \geq 0(i+j+\alpha \geq 2)$ be as before; then we have $c_{i, j, \alpha}(x) \ll A_{i, j, \alpha} /(R-x)$. We suppose: $K \geq 2$ and $0<R \leq 1$. We set

$$
G\left(t, x, w_{0}, w_{1}\right)=\sum_{i+j+\alpha \geq 2} \frac{A_{i, j, \alpha}}{(R-x)} t^{i} w_{0}^{j} w_{1}^{\alpha}+\frac{C_{K, 0}}{(R-x)} t^{K} .
$$

Let $\psi_{1}^{+}=w_{0}$ and

$$
\psi^{+}=w_{0}+\sum_{k \geq 2} \psi_{k}^{+}\left(t, x, w_{0}, \ldots, w_{k-1}\right) \in \sum_{k \geq 1} \mathcal{H}_{k,(R)}\left[t, w_{0}, \ldots, w_{k-1}\right]:
$$

let us consider the equation

$$
\left(\tau^{0}-1\right) \psi^{+}=G\left(t, x, \psi^{+}, D\left[\psi^{+}\right]\right), \quad \psi_{1}^{+}=w_{0},
$$

where

$$
\tau^{0}=t \frac{\partial}{\partial t}+\sum_{m \geq 0} w_{m} \frac{\partial}{\partial w_{m}}
$$

It is easy to see that this equation (4.8) has a unique formal solution of the form (4.7) and we have $\psi_{k}^{+}\left(t, x, w_{0}, \ldots, w_{k-1}\right) \gg 0$ (for $k \geq 2$ ): in particular we have

$$
\psi_{K}^{+}\left(t, x, w_{0}, \ldots, w_{K-1}\right) \gg \frac{1}{(K-1)} \frac{C_{K, 0}}{(R-x)} t^{K} .
$$

By the same argument as in Proposition 4.1 we have

Proposition 4.5. For any $r>0, c>0$ and $0<s<R$ we have

$$
\sum_{k \geq 1}\left\|\psi_{k}^{+}\right\|_{r, c, s} z^{k} \in \mathbf{C}\{z\}
$$

For $0<s \leq R$ and

$$
f=\sum_{i+|j|=k} f_{i, j}(x) t^{i} u_{0}{ }^{j_{0}} u_{1}{ }^{j_{1}} \cdots u_{k-1}{ }^{j_{k-1}} \in \mathcal{H}_{k, R}\left[t, u_{0}, \ldots, u_{k-1}\right]
$$


we write $|f|_{s}\left(t, u_{0}, \ldots, u_{k-1}\right)$ by

$$
|f|_{s}\left(t, u_{0}, \ldots, u_{k-1}\right)=\sum_{i+|j|=k}\left\|f_{i, j}\right\|_{s} t^{i} u_{0}{ }^{j_{0}} u_{1} j_{1} \cdots u_{k-1}^{j_{k-1}}
$$

We have

LEMMA 4.6. Let $\sigma_{0}>0$ be the one in (1.4). We can find constants $r_{0}>0$ and $0<c_{0}<R$ such that for any $0<r \leq r_{0}, 0<c \leq c_{0}, 0<s<R$ with $c+s=R$, and $0<\sigma \leq \sigma_{0} / 2$, we have the following results:

(1) If $k \geq 2$ and $k \neq K$, the term $\phi_{k}$ in the formal solution (4.1) satisfies

$$
\begin{gathered}
\sigma k\left|\phi_{k}\right|_{s}\left(r \rho, \psi^{+}\left(*_{\rho}\right), D\left[\psi^{+}\right]\left(*_{\rho}\right), \ldots, D^{k-1}\left[\psi^{+}\right]\left(*_{\rho}\right)\right) \\
\ll \rho \sum_{1 \leq q \leq k-1} \sum_{0 \leq m \leq q-1}\left|D^{m}\left[R^{(k-q+1)}\right]\right|_{s}\left(r \rho, \psi^{+}\left(*_{\rho}\right), \ldots, D^{m+1}\left[\psi^{+}\right]\left(*_{\rho}\right)\right) \\
\times \frac{\partial\left|\phi_{q}\right|_{s}}{\partial u_{m}}\left(r \rho, \psi^{+}\left(*_{\rho}\right), \ldots, D^{q-1}\left[\psi^{+}\right]\left(*_{\rho}\right)\right)
\end{gathered}
$$

where $\left(*_{\rho}\right)=\left(r \rho, s,\left(0 ! / c^{0}\right) \rho,\left(1 ! / c^{1}\right) \rho,\left(2 ! / c^{2}\right) \rho, \ldots\right)$.

(2) Let $\phi_{K}\left(t, x, u_{0}, \ldots, u_{K-1}\right)$ be the homogeneous part of degree $K$ of the formal solution (4.1), and set $\phi_{K}^{\circ}=\phi_{K}\left(t, x, u_{0}, \ldots, u_{K-1}\right)-\phi_{K, 0}(x) t^{K}$ : we have

$$
\begin{gathered}
\sigma K\left|\phi_{K}^{\circ}\right|_{s}\left(r \rho, \psi^{+}\left(*_{\rho}\right), D\left[\psi^{+}\right]\left(*_{\rho}\right), \ldots, D^{K-1}\left[\psi^{+}\right]\left(*_{\rho}\right)\right) \\
\ll_{\rho} \sum_{1 \leq q \leq K-1} \sum_{0 \leq m \leq q-1}\left|D^{m}\left[R^{(K-q+1)}\right]\right|_{s}\left(r \rho, \psi^{+}\left(*_{\rho}\right), \ldots, D^{m+1}\left[\psi^{+}\right]\left(*_{\rho}\right)\right) \\
\times \frac{\partial\left|\phi_{q}\right|_{s}}{\partial u_{m}}\left(r \rho, \psi^{+}\left(*_{\rho}\right), \ldots, D^{q-1}\left[\psi^{+}\right]\left(*_{\rho}\right)\right) .
\end{gathered}
$$

PROOF OF LEMMA 4.6. The proof of (1) is just the same as in [(2) of Lemma 3.4.5 in [15]] combined with $(3.4)_{k}$ (with $\gamma_{1}(x)$ replaced by $\left.\gamma(x)\right)$. Set

$$
\phi_{K}^{\circ}=\sum_{i+|j|=K,(i,|j|) \neq(K, 0)} \phi_{i, j}(x) t^{i} u_{0}{ }^{j_{0}} u_{1}{ }^{j_{1}} \cdots u_{K-1}{ }^{j_{K-1}} .
$$

We note: if $g\left(t, x, u_{0}, \ldots, u_{K-1}\right) \in \mathcal{H}_{k, R}\left[t, u_{0}, \ldots, u_{K-1}\right]$ satisfies

$$
\begin{aligned}
& \sum_{i+|j|=K,(i,|j|) \neq(K, 0)}(i+\lambda(x)(|j|-1)) \phi_{i, j}(x) t^{i} u_{0}{ }^{j_{0}} u_{1} j_{1} \cdots u_{K-1}{ }^{j_{K-1}} \\
& \equiv g\left(t, x, u_{0}, \ldots, u_{K-1}\right) \quad \bmod \mathcal{O}_{R} t^{K}
\end{aligned}
$$

(where $f \equiv g \bmod \mathcal{O}_{R} t^{K}$ means $f-g \in \mathcal{O}_{R} t^{K}$ ), by (1.4) we have

$$
\sigma_{0} K\left|\phi_{K}{ }^{\circ}\right|_{s}\left(t, u_{0}, \ldots, u_{K-1}\right) \ll|g|_{s}\left(t, u_{0}, \ldots, u_{K-1}\right) .
$$

By using this fact, let us show the part (2). 
Set

$$
\begin{aligned}
f_{K} & =-\sum_{1 \leq q \leq K-1} \sum_{0 \leq m \leq q-1} D^{m}\left[R^{(K-q+1)}\right]\left(t, x, u_{0}, \ldots, u_{m+1}\right) \frac{\partial \phi_{q}}{\partial u_{m}}, \\
\tau_{K, 1} & =t \frac{\partial}{\partial t}+\sum_{0 \leq m \leq K-1} \lambda(x) u_{m} \frac{\partial}{\partial u_{m}}, \\
\tau_{K, 2} & =\sum_{0 \leq m \leq K-1} a^{(m)}(x) t \frac{\partial}{\partial u_{m}}+\sum_{1 \leq m \leq K-1} \sum_{0 \leq p \leq m-1} \lambda_{m, p}(x) u_{p} \frac{\partial}{\partial u_{m}} .
\end{aligned}
$$

Then, we have $\tau_{K}=\tau_{K, 1}+\tau_{K, 2}$ and by (3.8) $K$ we have $\left(\tau_{K, 1}-\lambda(x)\right) \phi_{K}=-\tau_{K, 2} \phi_{K}+$ $\gamma(x) t^{K}+f_{K}$. Since $\phi_{K}=\phi_{K}{ }^{\circ}+\phi_{K, 0}(x) t^{K}$ holds, if we consider this equation in the modulo class $\mathcal{O}_{R} t^{K}$ we have $\left(\tau_{K, 1}-\lambda(x)\right) \phi_{K}{ }^{\circ}=-\left(\tau_{K, 1}-\lambda(x)\right)\left(\phi_{K, 0}(x) t^{K}\right)-\tau_{K, 2} \phi_{K}{ }^{\circ}-$ $\tau_{K, 2}\left(\phi_{K, 0}(x) t^{K}\right)+\gamma(x) t^{K}+f_{K}=-(K-\lambda(x))\left(\phi_{K, 0}(x) t^{K}\right)-\tau_{K, 2} \phi_{K}^{\circ}+\gamma(x) t^{K}+f_{K} \equiv$ $-\tau_{K, 2} \phi_{K}{ }^{\circ}+f_{K}$. Hence, we have

$$
\begin{aligned}
& \sum_{i+|j|=K,(i,|j|) \neq(K, 0)}(i+\lambda(x)(|j|-1)) \phi_{i, j}(x) t^{i} u_{0} j_{0} \cdots u_{K-1} j_{K-1} \\
& \equiv-\sum_{i+|j|=K,(i,|j|) \neq(K, 0)} \phi_{i, j}(x)\left[\sum_{0 \leq m \leq K-1} a^{(m)}(x) j_{m}\right. \\
& \times t^{i+1} u_{0} j_{0} \cdots u_{m}{ }^{j_{m}-1} \cdots u_{K-1} j_{K-1} \\
& \left.+\sum_{1 \leq m \leq K-1} \sum_{0 \leq p \leq m-1} \lambda_{m, p}(x) j_{m} t^{i} u_{0}^{j_{0}} \cdots u_{p}^{j_{p}+1} \cdots u_{m}{ }^{j_{m}-1} \cdots u_{K-1} j_{K-1}\right] \\
& +f_{K}\left(t, x, u_{0}, \ldots, u_{K-1}\right) \bmod \mathcal{O}_{R} t^{K} ;
\end{aligned}
$$

therefore, by (4.12) we have

$$
\begin{aligned}
& \sigma_{0} K\left|\phi_{K}^{\circ}\right|_{s}\left(t, u_{0}, \ldots, u_{K-1}\right) \\
& \ll \sum_{i+|j|=K,(i,|j|) \neq(K, 0)}\left\|\phi_{i, j}\right\|_{s}\left[\sum_{0 \leq m \leq K-1}\left\|a^{(m)}\right\|_{s} j_{m}\right. \\
& \times t^{i+1} u_{0}^{j_{0}} \cdots u_{m}^{j_{m}-1} \cdots u_{K-1}^{j_{K-1}} \\
& \left.+\sum_{1 \leq m \leq K-1} \sum_{0 \leq p \leq m-1}\left\|\lambda_{m, p}\right\|_{s} j_{m} t^{i} u_{0}^{j_{0}} \cdots u_{p}^{j_{p}+1} \cdots u_{m}^{j_{m}-1} \cdots u_{K-1}^{j_{K-1}}\right] \\
& +\left|f_{K}\right|_{s}\left(t, u_{0}, \ldots, u_{K-1}\right) \text {. }
\end{aligned}
$$

Thus, if we use this formula (4.13), by the same argument as in the proof of [Lemma 3.4.5 in [15]] we can show the existence of $r_{0}>0$ and $c_{0}>0$ such that

$$
\sigma_{0} K\left|\phi_{K}^{\circ}\right|_{s}\left(r \rho, \psi^{+}\left(*_{\rho}\right), \ldots, D^{K-1}\left[\psi^{+}\right]\left(*_{\rho}\right)\right)
$$




$$
\begin{aligned}
\ll_{\rho} \frac{\sigma_{0}}{2} K\left|\phi_{K}^{\circ}\right|_{s}\left(r \rho, \psi^{+}\left(*_{\rho}\right), \ldots, D^{K-1}\left[\psi^{+}\right]\left(*_{\rho}\right)\right) \\
+\left|f_{K}\right|_{s}\left(r \rho, \psi^{+}\left(*_{\rho}\right), \ldots, D^{K-1}\left[\psi^{+}\right]\left(*_{\rho}\right)\right)
\end{aligned}
$$

holds for any $0<r \leq r_{0}, 0<c \leq c_{0}$, and $0<s<R$ with $c+s=R$. Thus we obtain the inequality

$$
\begin{aligned}
& \frac{\sigma_{0}}{2} K\left|\phi_{K}^{\circ}\right|_{s}\left(r \rho, \psi^{+}\left(*_{\rho}\right), \ldots, D^{K-1}\left[\psi^{+}\right]\left(*_{\rho}\right)\right) \\
& \ll_{\rho}\left|f_{K}\right|_{s}\left(r \rho, \psi^{+}\left(*_{\rho}\right), \ldots, D^{K-1}\left[\psi^{+}\right]\left(*_{\rho}\right)\right) \\
& \ll_{\rho} \sum_{1 \leq q \leq K-1} \sum_{0 \leq m \leq q-1}\left|D^{m}\left[R^{(K-q+1)}\right]\right|_{S}\left(r \rho, \psi^{+}\left(*_{\rho}\right), \ldots, D^{m+1}\left[\psi^{+}\right]\left(*_{\rho}\right)\right) \\
& \times \frac{\partial\left|\phi_{q}\right|_{s}}{\partial u_{m}}\left(r \rho, \psi^{+}\left(*_{\rho}\right), \ldots, D^{q-1}\left[\psi^{+}\right]\left(*_{\rho}\right)\right) .
\end{aligned}
$$

This proves (2) of Lemma 4.6.

Take any $0<\sigma \leq \min \left\{\sigma_{0} / 2,1\right\}$ and fix it: let us take $\mu>0$ such that

$$
\mu \geq \frac{\sigma^{K-1}(K-1) ! \times(K-1)}{(K-1)^{K-1}} .
$$

We have

LEMMA 4.7. Let $r_{0}>0$ and $0<c_{0}<R$ be as in Lemma 4.6. Then, for any $0<r \leq$ $r_{0}, 0<c \leq c_{0}, 0<s<R$ with $c+s=R$ we have the following results:

(1) If $k=1$ we have $\phi_{1}=u_{0}-\alpha(x) t$ and so

$$
\left|\phi_{1}\right|_{s}\left(r \rho, \psi^{+}\left(*_{\rho}\right)\right) \ll_{\rho}\|\alpha\|_{s} r \rho+\psi_{1}^{+}(*) \rho+\psi_{2}^{+}(*) \rho^{2}+\psi_{3}^{+}(*) \rho^{3}+\cdots
$$

where $(*)=\left(r, s, 0 ! / c^{0}, 1 ! / c^{1}, 2 ! / c^{2}, \ldots\right)$.

(2) If $k \geq 2$ we have

$$
\begin{gathered}
\sum_{1 \leq q \leq k-1} \sum_{0 \leq m \leq q-1} D^{m}[G]\left(r \rho, s, \psi^{+}\left(*_{\rho}\right), \ldots, D^{m+1}\left[\psi^{+}\right]\left(*_{\rho}\right)\right) \\
\times \frac{\partial\left|\phi_{q}\right|_{s}}{\partial u_{m}}\left(r \rho, \psi^{+}\left(*_{\rho}\right), \ldots, D^{q-1}\left[\psi^{+}\right]\left(*_{\rho}\right)\right) \\
\ll M_{k} \sum_{2 \leq p \leq k-1} \frac{(p-1)^{p-1}}{\sigma^{p-2}(p-2) !} \psi_{p}^{+}(*) \rho^{p}+M_{k} \sum_{p \geq k} \frac{(p-1)^{k-1}}{\sigma^{k-2}(k-2) !} \psi_{p}^{+}(*) \rho^{p}
\end{gathered}
$$

where $M_{k}=1$ for $2 \leq k \leq K$, and $M_{k}=1+\mu$ for $k \geq K+1$.

(3) If $k \geq 2$ we have

$$
\left|\phi_{k}\right|_{s}\left(r \rho, \psi^{+}\left(*_{\rho}\right), \ldots, D^{k-1}\left[\psi^{+}\right]\left(*_{\rho}\right)\right) \ll_{\rho} L_{k} \sum_{p \geq k} \frac{(p-1)^{k-1}}{\sigma^{k-1}(k-1) !} \psi_{p}^{+}(*) \rho^{p}
$$


where $L_{k}=1$ for $2 \leq k \leq K-1$, and $L_{k}=1+\mu$ for $k \geq K$.

PROOF OF LEMMA 4.7. The proofs of (1), (4.15) $k$ (for $2 \leq k \leq K$ ), and (4.16) $k$ (for $2 \leq k \leq K-1)$ are the same as in [Proposition 3.4.8 in [15]].

Let us show $(4.16)_{K}$. Since $\phi_{K}{ }^{\circ}\left(t, x, u_{0}, \ldots, u_{K-1}\right)$ is homogeneous of degree $K$ in $\left(t, u_{0}, \ldots, u_{K-1}\right)$ and since $R^{(K-q+1)}\left(t, x, w_{0}, w_{1}\right) \ll G\left(t, x, w_{0}, w_{1}\right)$ holds, by $(4.15)_{K}$ and (2) of Lemma 4.6 we have

$$
\left|\phi_{K}^{\circ}\right|_{s}\left(r \rho, \psi^{+}\left(*_{\rho}\right), \ldots, D^{K-1}\left[\psi^{+}\right]\left(*_{\rho}\right)\right) \ll_{\rho} \sum_{p \geq K} \frac{(p-1)^{K-1}}{\sigma^{K-1}(K-1) !} \psi_{p}^{+}(*) \rho^{p} .
$$

Since $\phi_{K}=\phi_{K}{ }^{\circ}+\psi_{K, 0}(x) t^{K}$, by (4.5) we have

$$
\begin{aligned}
\left|\phi_{K}\right|_{s} & \left(r \rho, \psi^{+}\left(*_{\rho}\right), \ldots, D^{K-1}\left[\psi^{+}\right]\left(*_{\rho}\right)\right) \\
& \ll_{\rho}\left|\phi_{K}\right|_{s}\left(r \rho, \psi^{+}\left(*_{\rho}\right), \ldots, D^{K-1}\left[\psi^{+}\right]\left(*_{\rho}\right)\right)+\left\|\psi_{K, 0}\right\|_{s}(r \rho)^{K} \\
& \ll_{\rho} \sum_{p \geq K} \frac{(p-1)^{K-1}}{\sigma^{K-1}(K-1) !} \psi_{p}^{+}(*) \rho^{p}+\frac{C_{K, 0}}{(R-s)}(r \rho)^{K} .
\end{aligned}
$$

Here we note that by (4.9) and (4.14) we have

$$
\frac{C_{K, 0}}{(R-s)}(r \rho)^{K} \ll_{\rho}(K-1) \psi_{K}(*) \rho^{K} \ll_{\rho} \mu \times \frac{(K-1)^{K-1}}{\sigma^{K-1}(K-1) !} \psi_{K}(*) \rho^{K} .
$$

Thus, by applying this to (4.17) we obtain the result $(4.16)_{K}$.

If $k \geq K+1$, we can use (1) of Lemma 4.6 and so we can prove $(4.15)_{k}$ and (4.16) $k$ in the same way, by induction on $k$.

Now, let us complete the proof of Proposition 4.4. By comparing the homogeneous part of degree $k$ with respect to $\rho$ in the both sides of $(4.16)_{k}$, we have

$$
\left|\phi_{k}\right|_{s}\left(r, \psi_{1}^{+}(*), \ldots, D^{k-1}\left[\psi_{1}^{+}\right](*)\right) \leq(1+\mu) \frac{(k-1)^{k-1}}{\sigma^{k-1}(k-1) !} \psi_{k}^{+}(*)
$$

for any $k \geq 2$. Since $D^{j}\left[\psi_{1}^{+}\right](*)=\left(D^{j}\left[w_{0}\right]\right)(*)=\left(w_{j}\right)(*)=j ! / c^{j}$ holds for all $j \in \mathbf{N}$, we obtain

$$
\left|\phi_{k}\right|_{s}\left(r, \frac{0 !}{c^{0}}, \frac{1 !}{c^{1}}, \ldots, \frac{(k-1) !}{c^{k-1}}\right) \leq(1+\mu) \frac{(k-1)^{k-1}}{\sigma^{k-1}(k-1) !} \psi_{k}^{+}\left(r, s, \frac{0 !}{c^{0}}, \frac{1 !}{c^{1}}, \ldots, \frac{(k-1) !}{c^{k-1}}\right):
$$

this is equivalent to

$$
\left\|\phi_{k}\right\|_{r, c, s} \leq(1+\mu) \frac{(k-1)^{k-1}}{\sigma^{k-1}(k-1) !}\left\|\psi_{k}^{+}\right\|_{r, c, s}, \quad k \geq 2 .
$$

Thus, by Proposition 4.5 and the Stirling's formula we have the result (4.6). 


\section{Completion of the proof of Theorem 1.2}

For $r>0, c>0, s>0$ and $\varepsilon>0$ we write

$$
\begin{array}{r}
U_{k}(r, c, s, \varepsilon)=\left\{\left(t, x, u_{0}, \ldots, u_{k-1}\right) \in \mathbf{C} \times \mathbf{C} \times \mathbf{C}^{k} ;|t| \leq \varepsilon r,|x| \leq s,\right. \\
\left.\left|u_{0}\right| \leq 0 ! \varepsilon,\left|u_{1}\right| \leq 1 ! \varepsilon / c, \ldots,\left|u_{k-1}\right| \leq(k-1) ! \varepsilon / c^{k-1}\right\}, \\
W_{k}(r, c, s, \varepsilon)=\left\{\left(t, x, w_{0}, \ldots, w_{k-1}\right) \in \mathbf{C} \times \mathbf{C} \times \mathbf{C}^{k} ;|t| \leq \varepsilon r,|x| \leq s,\right. \\
\left.\left|w_{0}\right| \leq 0 ! \varepsilon,\left|w_{1}\right| \leq 1 ! \varepsilon / c, \ldots,\left|w_{k-1}\right| \leq(k-1) ! \varepsilon / c^{k-1}\right\}
\end{array}
$$

$(k=1,2, \ldots)$. For a function $f\left(t, x, u_{0}, u_{1}, \ldots, u_{k-1}\right)$ in a neighborhood of $U_{k}=$ $U_{k}(r, c, s, \varepsilon)$ we write

$$
\|f\|_{U_{k}}=\max _{U_{k}}\left|f\left(t, x, u_{0}, u_{1}, \ldots, u_{k-1}\right)\right| .
$$

The norm $\|g\|_{W_{k}}$ may be defined in the same way. Then, by the results in $\S 4$ we have:

Proposition 5.1. Suppose the conditions $\left.\left.\left.\mathrm{A}_{1}\right), \mathrm{A}_{2}\right), \mathrm{A}_{3}\right)$ and $\lambda(0)=K \in\{1,2, \ldots\}$. Let

$$
\phi=\sum_{k \geq 1} \phi_{k}\left(t, x, u_{0}, \ldots, u_{k-1}\right) \quad \text { and } \quad \psi=\sum_{k \geq 1} \psi_{k}\left(t, x, w_{0}, \ldots, w_{k-1}\right)
$$

be the formal solutions in Proposition 3.9. Then, we can find constants $r_{0}>0$ and $0<c_{0}<$ $R$ so that the following results hold.

(1) For any $0<r \leq r_{0}, 0<c \leq c_{0}$ and $s=R-c$ there is an $\varepsilon>0$ such that the series

$$
\sum_{k \geq 1}\left\|\phi_{k}\right\|_{U_{k}} \text { with } U_{k}=U_{k}(r, c, s, \varepsilon)
$$

is convergent.

(2) For any $r>0,0<c \leq c_{0}$ and $0<s<R$ there is an $\varepsilon>0$ such that the series

$$
\sum_{k \geq 1}\left\|\psi_{k}\right\|_{W_{k}} \quad \text { with } W_{k}=W_{k}(r, c, s, \varepsilon)
$$

is convergent.

By this result we see: if a holomorphic function $u(t, x)$ (resp. $w(t, x))$ satisfies $\left|(\partial / \partial x)^{k} u(t, x)\right| \leq k ! \varepsilon / c^{k}$ for $k=0,1,2, \ldots\left(\operatorname{resp} .\left|(\partial / \partial x)^{k} w(t, x)\right| \leq k ! \varepsilon / c^{k}\right.$ for $k=0,1,2, \ldots)$, we can define the function $w(t, x)=\phi(t, x, u, \partial u / \partial x, \ldots)($ resp. $u(t, x)=$ $\psi(t, x, w, \partial w / \partial x, \ldots))$. Let us introduce suitable classes of such holomorphic functions. For simplicity, we will use the following symbols:

$$
\begin{aligned}
& \text { - } S_{\theta}=\{t \in \mathcal{R}(\mathbf{C} \backslash\{0\}) ;|\arg t|<\theta\} \text { a sector in } \mathcal{R}(\mathbf{C} \backslash\{0\}), \\
& \text { - } S_{\theta}(r)=\{t \in \mathcal{R}(\mathbf{C} \backslash\{0\}) ;|\arg t|<\theta, 0<|t| \leq r\},
\end{aligned}
$$


- $S(\varepsilon(s))=\{t \in \mathcal{R}(\mathbf{C} \backslash\{0\}) ; 0<|t| \leq \varepsilon(\arg t)\}$, where $\varepsilon(s)$ is a positivevalued continuous function on $\mathbf{R}_{s}$.

The function spaces $\mathscr{S}_{+}$and $\mathscr{S}_{\text {zero }}$ are already defined in $\S 1$; though, we will repeat them so that we can easily compare these with other spaces.

DEFINITION 5.2. (1) We denote by $\mathcal{O}_{+}$the set of all holomorphic functions $u(t, x)$ in a neighborhood of $(0,0) \in \mathbf{C}^{2}$ satisfying $u(0, x) \equiv 0$.

(2) We denote by $\widetilde{\mathcal{O}}_{+}$the set of all $u(t, x)$ satisfying the following i) and ii): i) $u(t, x)$ is a holomorphic function on $S(\varepsilon(s)) \times D_{R}$ for some positive-valued continuous function $\varepsilon(s)$ on $\mathbf{R}_{s}$ and $R>0$; and ii) there is an $a>0$ such that for any $\theta>0$ we have

$$
\max _{x \in D_{R}}|u(t, x)|=O\left(|t|^{a}\right) \quad\left(\text { as } t \longrightarrow 0 \text { in } S_{\theta}\right) .
$$

(3) We denote by $\mathscr{S}_{+}$the set of all $u(t, x)$ satisfying the following i) and ii): i) $u(t, x)$ is a holomorphic function on $S_{\theta}(r) \times D_{R}$ for some $\theta>0, r>0$ and $R>0$; and ii) there is an $a>0$ such that

$$
\max _{x \in D_{R}}|u(t, x)|=O\left(|t|^{a}\right) \quad\left(\text { as } t \longrightarrow 0 \text { in } S_{\theta}(r)\right) .
$$

(4) We denote by $\mathscr{S}_{\text {zero }}$ the set of all $u(t, x)$ satisfying the following i) and ii): i) $u(t, x)$ is a holomorphic function on $S_{\theta}(r) \times D_{R}$ for some $\theta>0, r>0$ and $R>0$; and ii) we have

$$
\max _{x \in D_{R}}|u(t, x)|=o(1) \quad\left(\text { as } t \longrightarrow 0 \text { in } S_{\theta}(r)\right) .
$$

Then, by Proposition 5.1 we can see:

Proposition 5.3. Set $\mathcal{F}=\mathcal{O}_{+}, \widetilde{\mathcal{O}}_{+}, \mathscr{S}_{+}$or $\mathscr{S}_{\text {zero. }}$ The following two mappings are well-defined:

$$
\begin{aligned}
& \Phi: \mathcal{F} \ni u(t, x) \longmapsto w(t, x)=\phi(t, x, u, \partial u / \partial x, \ldots) \in \mathcal{F}, \\
& \Psi: \mathcal{F} \ni w(t, x) \longmapsto u(t, x)=\psi(t, x, w, \partial w / \partial x, \ldots) \in \mathcal{F} .
\end{aligned}
$$

By combining this with Theorem 3.10 we obtain

THEOREM 5.4. Set $\mathcal{F}=\mathcal{O}_{+}, \widetilde{\mathcal{O}}_{+}, \mathscr{S}_{+}$or $\mathscr{S}_{\text {zero. }}$ Suppose the conditions $\left.\left.\mathrm{A}_{1}\right), \mathrm{A}_{2}\right)$, $\left.\mathrm{A}_{3}\right)$ and $\lambda(0)=K \in\{1,2, \ldots\}$. Then, there is a holomorphic function $\mu(x) \in \mathcal{O}_{x}$ such that for any $\gamma(x) \in\left(\mathcal{O}_{x}(K-\lambda(x))+\mu(x)\right)$ the following two equations are equivalent:

$$
\begin{aligned}
& t \frac{\partial u}{\partial t}=F\left(t, x, u, \frac{\partial u}{\partial x}\right) \quad \text { in } \mathcal{F}, \\
& t \frac{\partial w}{\partial t}=\lambda(x) w+\gamma(x) t^{K} \quad \text { in } \mathcal{F} .
\end{aligned}
$$




\section{Application}

As an application, let us determine all the holomorphic and singular solutions of (5.3). Since (5.3) is equivalent to the reduced form (5.4), we have only to determine all the holomorphic and singular solutions of (5.4).

6.1. Singular solutions. Let us denote by $\mathscr{S}_{*}$ the set of all holomorphic functions $u(t, x)$ on $S_{\theta}(r) \times D_{R}$ for some $\theta>0, r>0$ and $R>0$ (which may depend on $u(t, x)$ ). We have

$$
\mathcal{O}_{+} \subset \mathscr{S}_{+} \subset \mathscr{S}_{\text {zero }} \subset \mathscr{S}_{*}
$$

By the integration we see that the set $\operatorname{Sol}\left((6.1), \mathscr{S}_{*}\right)$ of all the solutions of the equation

$$
t \frac{\partial w}{\partial t}=\lambda(x) w+\gamma(x) t^{K} \quad \text { in } \mathscr{S}_{*}
$$

is given by

$$
\operatorname{Sol}\left((6.1), \mathscr{S}_{*}\right)=\left\{\varphi(x) t^{\lambda(x)}+\gamma(x) H(t, x) ; \varphi(x) \in \mathcal{O}_{x}\right\},
$$

where $H(t, x)$ is a solution of $(t \partial / \partial t-\lambda(x)) H=t^{K}$ defined by

$$
H(t, x)=\frac{t^{\lambda(x)}-t^{K}}{\lambda(x)-K}=t^{K} \times \frac{t^{\lambda(x)-K}-1}{\lambda(x)-K}=t^{K} \sum_{p \geq 1} \frac{(\lambda(x)-K)^{p-1}}{p !}(\log t)^{p} .
$$

In the case $\lambda(x) \equiv K$, by the last equality we have $H(t, x)=t^{K} \log t$.

Let us show that $H(t, x) \in \widetilde{\mathcal{O}}_{+}$holds. Take any $0<a<1$; since $\lambda(0)=K$ is supposed, we have $|\lambda(x)-K| \leq a$ on $D_{R}$ for some $R>0$. Then, if $|t|<1$ and $x \in D_{R}$ we have

$$
\begin{aligned}
|H(t, x)| & \leq|t|^{K} \sum_{p \geq 1} \frac{a^{p-1}}{p !}(-\log |t|)^{p} \leq|t|^{K} \sum_{p \geq 1} \frac{a^{p-1}}{(p-1) !}(-\log |t|)^{p} \\
& =|t|^{K}(-\log |t|) e^{-a \log |t|}=|t|^{K-a}(-\log |t|) .
\end{aligned}
$$

Since $K \geq 1$ we have $K-a>0$ and so $H(t, x) \in \widetilde{\mathcal{O}}_{+}$. This shows:

Proposition 6.1. We denote by $\operatorname{Sol}((6.1), \mathcal{F})$ the set of all solutions of $(6.1)$ with $\mathscr{S}_{*}$ replaced by $\mathcal{F}$. Then we have

$$
\begin{aligned}
\operatorname{Sol}\left((6.1), \widetilde{\mathcal{O}}_{+}\right) & =\operatorname{Sol}\left((6.1), \mathscr{S}_{+}\right)=\operatorname{Sol}\left((6.1), \mathscr{S}_{\text {zero }}\right) \\
& =\left\{\varphi(x) t^{\lambda(x)}+\gamma(x) H(t, x) ; \varphi(x) \in \mathcal{O}_{x}\right\} .
\end{aligned}
$$

Thus, we have

THEOREM 6.2. Suppose the conditions $\left.\left.\left.\mathrm{A}_{1}\right), \mathrm{A}_{2}\right), \mathrm{A}_{3}\right)$ and $\lambda(0)=K \in\{1,2, \ldots\}$. Denote by $\operatorname{Sol}((5.3), \mathcal{F})$ the set of all solutions of $(5.3)$. Then we have

$$
\operatorname{Sol}\left((5.3), \widetilde{\mathcal{O}}_{+}\right)=\operatorname{Sol}\left((5.3), \mathscr{S}_{+}\right)=\operatorname{Sol}\left((5.3), \mathscr{S}_{\text {zero }}\right)
$$




$$
=\left\{\Psi\left[\varphi(x) t^{\lambda(x)}+\gamma(x) H(t, x)\right] ; \varphi(x) \in \mathcal{O}_{x}\right\} .
$$

Let us see the series form of $\Psi\left[\varphi(x) t^{\lambda(x)}+\gamma(x) H(t, x)\right]$. By Proposition 3.9 we know that $\psi\left(t, x, w_{0}, w_{1}, \ldots\right)$ has the form

$$
\psi=w_{0}+\alpha(x) t+\sum_{k \geq 2} \psi_{k}\left(t, x, w_{0}, \ldots, w_{k-1}\right)
$$

and each $\psi_{k}$ (for $k \geq 2$ ) has the form (3.12) in Proposition 3.4. Hence, we have

Corollary 6.3. Suppose the conditions $\left.\left.\left.\mathrm{A}_{1}\right), \mathrm{A}_{2}\right), \mathrm{A}_{3}\right)$ and $\lambda(0)=K \in\{1,2, \ldots\}$. Set $G(t, x)=t^{\lambda(x)}$ and let $H(t, x)$ be the one in (6.2). Then, every solution $u(t, x) \in$ $\operatorname{Sol}\left((5.3), \widetilde{\mathcal{O}}_{+}\right)\left(=\operatorname{Sol}\left((5.3), \mathscr{S}_{+}\right)=\operatorname{Sol}\left((5.3), \mathscr{S}_{\text {zero }}\right)\right)$ is expanded into the form

$$
\begin{aligned}
& u(t, x)=\Psi[\varphi(x) G+\gamma(x) H] \\
& =\varphi(x) G+\gamma(x) H+\sum_{k \geq 1} \psi_{k, 0}(x) t^{k} \\
& +\sum_{k \geq 2} \sum_{\begin{array}{c}
i+|p|+|q|=k,|p|+|q| \geq 1 \\
i+p_{0}+q_{0}+2\left(\left|p^{\prime}\right|+\left|q^{\prime}\right|-1\right) \geq\langle p\rangle+\langle q\rangle
\end{array}} \varphi_{i, p, q}(x) t^{i} \prod_{\substack{0 \leq \alpha \leq k-1 \\
0 \leq \beta \leq k-1}}\left(G^{(\alpha)}\right)^{p_{\alpha}}\left(H^{(\beta)}\right)^{q_{\beta}}
\end{aligned}
$$

for some holomorphic functions $\varphi_{i, p, q}(x) \in \mathcal{O}_{R}(i+|p|+|q|=k,|p|+|q| \geq 1, i+$ $p_{0}+q_{0}+2\left(\left|p^{\prime}\right|+\left|q^{\prime}\right|-1\right) \geq\langle p\rangle+\langle q\rangle$ and $\left.k \geq 2\right)$ with $R>0$ sufficiently small, where $p=\left(p_{0}, p_{1}, \ldots, p_{k-1}\right), q=\left(q_{0}, q_{1}, \ldots, q_{k-1}\right),|p|=p_{0}+p_{1}+\cdots+p_{k-1},|q|=q_{0}+q_{1}+$ $\cdots+q_{k-1},\left|p^{\prime}\right|=p_{1}+\cdots+p_{k-1},\left|q^{\prime}\right|=q_{1}+\cdots+q_{k-1},\langle p\rangle=p_{1}+2 p_{2}+\cdots+(k-1) p_{k-1}$, $\langle q\rangle=q_{1}+2 q_{2}+\cdots+(k-1) q_{k-1}, G^{(\alpha)}=(\partial / \partial x)^{\alpha} G$ and $H^{(\beta)}=(\partial / \partial x)^{\beta} H$.

REMARK 6.4. In the case $\mathcal{F}=\widetilde{\mathcal{O}}_{+}$or $\mathscr{S}_{+}$, Theorem 6.2 and Corollary 6.3 were obtained by Yamazawa [16].

6.2. Holomorphic solutions. Recall that we have taken $\mu(x)=\mu_{2}(x)$; therefore, if $K=1$ we have $\mu(x)=a(x)$, and if $K \geq 2$ we have (by (3.11))

$$
\mu(x)=\sum_{2 \leq i+j+\alpha \leq K} c_{i, j, \alpha}(x)\left[\sum_{|p(j)|+|q(\alpha)|=K-i} \psi_{p_{1}, 0} \cdots \psi_{p_{j}, 0} \frac{\partial \psi_{q_{1}, 0}}{\partial x} \cdots \frac{\partial \psi_{q_{\alpha}, 0}}{\partial x}\right],
$$

where $\psi_{q, 0}(x)$ is the coefficient of $t^{q}$ in the solution $\psi\left(t, x, w_{0}, w_{2}, \ldots\right)$ of $(\Psi),|p(j)|=$ $p_{1}+\cdots+p_{j}$ and $|q(\alpha)|=q_{1}+\cdots+q_{\alpha}$.

Let us divide our situation into the following four cases:

(i) $\lambda(x) \equiv K$ and $\mu(x) \equiv 0$,

(ii) $\lambda(x) \equiv K$ and $\mu(x) \not \equiv 0$,

(iii) $\lambda(x) \not \equiv K$ and $\mu(x) /(K-\lambda(x))$ is holomorphic at $x=0$,

(iv) $\lambda(x) \not \equiv K$ and $\mu(x) /(K-\lambda(x))$ is not holomorphic at $x=0$.

Then we have 
LEMMA 6.5. In the above situation we have: in the case (i) we can take $\gamma(x) \equiv 0$, in the case (ii) we can take $\gamma(x)=\mu(x)$, in the case (iii) we can take $\gamma(x) \equiv 0$, and in the case (iv) we can take $\gamma(x)=\mu(x)$.

PROOF. We recall that $\gamma(x)$ is taken so that $\gamma(x)=(K-\lambda(x)) f(x)+\mu(x)$ holds and $f(x) \in \mathcal{O}_{x}$ can be taken arbitrarily. Therefore, in the cases (i), (ii) and (iv) we take $f(x)=0$; then we have $\gamma(x)=\mu(x)$. In the case (iii) we take $f(x)=-\mu(x) /(K-\lambda(x))$; then we have $\gamma(x)=0$.

We already know that two equations (5.3) and (5.4) are equivalent. Therefore, to see the structure of $\operatorname{Sol}\left((5.3), \mathcal{O}_{+}\right)$it is enough to consider

$$
t \frac{\partial w}{\partial t}=\lambda(x) w+\gamma(x) t^{K} \quad \text { in } \mathcal{O}_{+}
$$

with $\gamma(x)$ being taken as in Lemma 6.5. Then, by an easy calculation we have the following result: in the case (i) we have $\operatorname{Sol}\left((6.7), \mathcal{O}_{+}\right)=\left\{\varphi(x) t^{K} ; \varphi(x) \in \mathcal{O}_{x}\right\}$, in the case (ii) we have $\operatorname{Sol}\left((6.7), \mathcal{O}_{+}\right)=\emptyset$, in the case (iii) we have $\operatorname{Sol}\left(\left(6.7, \mathcal{O}_{+}\right)\right)=\{0\}$, and in the case (iv) we have $\operatorname{Sol}\left((6.7), \mathcal{O}_{+}\right)=\emptyset$. This leads us to

THEOREM 6.6. Suppose $\left.\left.\left.\mathrm{A}_{1}\right), \mathrm{A}_{2}\right), \mathrm{A}_{3}\right)$ and $\lambda(0)=K \in\{1,2, \ldots\}$. Then, we have: in the case (i) the equation (1.1) has infinitely many holomorphic solutions satisfying $u(0, x) \equiv$ 0 , in the case (ii) the equation (1.1) has no holomorphic solutions satisfying $u(0, x) \equiv 0$, in the case (iii) the equation (1.1) has a unique holomorphic solution satisfying $u(0, x) \equiv 0$, and in the case (iv) the equation (1.1) has no holomorphic solutions satisfying $u(0, x) \equiv 0$.

\section{References}

[ 1 ] V. I. ARNOLD, Geometrical methods in the theory of ordinary differential equations, Grundlehren der Mathematischen Wissenschaften, 250. Springer-Verlag, New York-Berlin, 1983.

[2] M. S. Baouendi and C. Goulaouic, Singular nonlinear Cauchy problems, J. Differential Equations 22 (1976), no. 2, 268-291.

[ 3 ] J.-E. BJÖRK, Analytic $\mathscr{D}$-modules and applications, Mathematics and its Applications, 247. Kluwer Academic Publishers Group, Dordrecht, 1993.

[4] CH. BRIOT and J. CL. BouQuet, Recherches sur les proprietes des fonctions defmies par des equations differentielles, J. Ecole Polytech. 21 (1856), 133-197.

[ 5 ] H. Chen, Z. LUO and H. TAHARA, Formal solutions of nonlinear first order totally characteristic type pde with irregular singularity, Ann. Inst. Fourier (Grenoble) 51 (2001), no. 6, 1599-1620.

[ 6] H. CHEN, Z. LuO and C. ZHANG, On the summability of formal solutions for a class of nonlinear singular PDEs with irregular singularity, Recent progress on some problems in several complex variables and partial differential equations, 53-64, Contemp. Math., 400, Amer. Math. Soc., Providence, RI, 2006.

[ 7 ] H. Chen and H. TAHARA, On totally characteristic type non-linear partial differential equations in the complex domain, Publ. Res. Inst. Math. Sci. 35 (1999), no. 4, 621-636.

[ 8 ] R. GÉRARD, Etude locale des equations differentielles de la forme $x y^{\prime}=f(x, y)$ au voisinage de $x=0$, J. Fac. Sci. Univ. Tokyo Sect. IA, Math. 36 (1989), 729-752.

[9] R. GÉRARD and H. TAHARA, Singular nonlinear partial differential equations, Aspects of Mathematics. Friedr. Vieweg, Braunschweig, 1996. 
[10] R. GÉRARD and H. TAHARA, Holomorphic and singular solutions of nonlinear singular first order partial differential equations, Publ. Res. Inst. Math. Sci. 26 (1990), no. 6, 979-1000.

[11] E. HiLlE, Ordinary differential equations in the complex domain, John Wiley and Sons, 1976.

[12] J. E. C. LOPE, M. P. RoQUE and H. TAHARA, On the unique solvability of certain nonlinear singular partial differential equations, Z. Anal. Anwend. 31 (2012), no. 3, 291-305.

[13] Z. LUO, H. CHEN and C. ZHANG, Exponential-type Nagumo norms and summabolity of formal solutions of singular partial differential equations, Ann. Inst. Fourier, 62 (2012), no. 2, 571-618

[14] H. Tahara, Coupling of two partial differential equations and its application, Publ. Res. Inst. Math. Sci. 43 (2007), no. 3, 535-583.

[15] H. TAhara, Coupling of two partial differential equations and its application II, -the case of Briot-Bouquet type PDEs-, Publ. Res. Inst. Math. Sci. 45 (2009), no. 2, 393-449.

[16] H. Yamazawa, Singular solutions of the Briot-Bouquet type partial differential equations, J. Math. Soc. Japan 55 (2003), no. 3, 617-632.

[17] M. Yoshino, WKB analysis and Poincare theorem for vector fields, Algebraic analysis of differential equations from microlocal analysis to exponential asymptotics, 335-352, Springer, Tokyo, 2008.

Present Address:

DEPARTMENT OF INFORMATION AND COMMUNICATION SCIENCES,

SOPHIA UNIVERSITY,

KIOICHO, CHIYODA-KU, TOKYO, 102-8554 JAPAN.

e-mail: h-tahara@hoffman.cc.sophia.ac.jp 